\title{
EXCHANGE EFFECTS AND CIDEP
}

\author{
WILLIAM S. JENKS AND NICHOLAS J. TURRO* \\ Department of Chemistry \\ Columbia University \\ New York, NY 10027, USA
}

\section{CONTENTS}

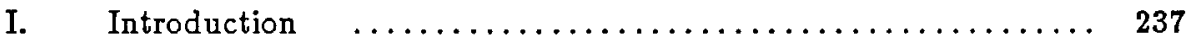

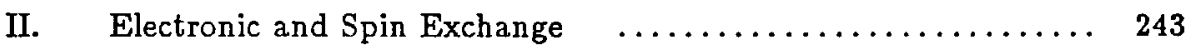

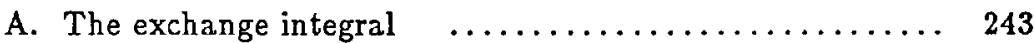

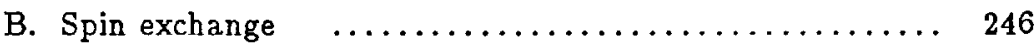

III. Experimental Exchange Phenomena $\ldots \ldots \ldots \ldots \ldots \ldots \ldots \ldots . \ldots . \ldots . \ldots . \ldots 249$

A. Radical pair mechanism and spin exchange $\ldots \ldots \ldots .249$

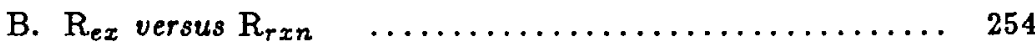

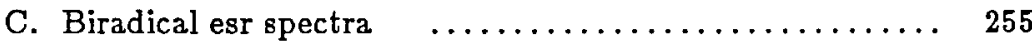

D. Biradical and correlated radical pair CIDEP $\ldots \ldots \ldots 261$

E. Spin exchange induced intersystem crossing $\ldots \ldots \ldots 266$

F. Energy transfer, electron transfer, and CIDEP $\ldots \ldots \ldots 271$

G. Late time phase inversion in CIDEP $\ldots \ldots \ldots \ldots \ldots \ldots 279$

IV. Spin Exchange versus Reaction: Spin Polarization Transfer in Doublet-Doublet Systems $\ldots \ldots \ldots \ldots \ldots \ldots \ldots \ldots \ldots .280$

A. Fluorenylidene/9-fluorenyl system: hydrogen

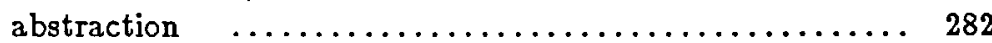

B. Xanthone system: electron transfer $\ldots \ldots \ldots \ldots \ldots \ldots 285$

C. Benzil dimethyl monoketal (BDM) system:

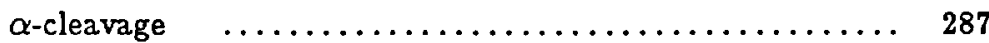

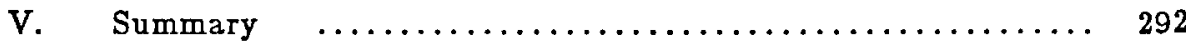

VI. Appendix: Experimental Section $\ldots \ldots \ldots \ldots \ldots \ldots \ldots \ldots . \ldots . \ldots 29$

VII. Acknowledgements $\ldots \ldots \ldots \ldots \ldots \ldots \ldots \ldots \ldots \ldots \ldots \ldots \ldots . \ldots \ldots$

VIII. References $\ldots \ldots \ldots \ldots \ldots \ldots \ldots \ldots \ldots \ldots \ldots \ldots \ldots \ldots \ldots \ldots . \ldots 294$

\section{INTRODUCTION}

Electron exchange interactions are involved in a great variety of chemical phenomena, from energy transfer to CIDEP to spin exchange and 
more. In this review, we hope to tie these and some other ideas together and to show some of their relationships. Most of the broad topics have been reviewed before, but each is briefly developed here in the interest of "leveling the playing field". We hope to reach an audience of nonspecialists whose interest (like ours) overlap into at least some of the topics covered here.

The starting point for any discussion of exchange interactions must be the quantum mechanical expression for the exchange integral

$$
K=<\phi_{a}(1) \phi_{b}(2)\left|e^{2} / r_{12}\right| \phi_{b}(1) \phi_{a}(2)>
$$

where $\phi_{a}(1)$ represents electron 1 in orbital a, and so on, and $r_{12}$ is the interelectron distance. Most of the phenomena we will discuss below which are called "exchange phenomena" in the literature also involve other energy terms, such as Coulomb, dipole, and overlap interactions. Some of the ambiguities in the literature nomenclature are addressed in the next section, and the effects of "exchange" energy differences on various phenomena, such as energy transfer and biradical esr and spin polarization, are examined in section III.

We will see that the so-called exchange energy is also central to Heisenberg spin exchange. Spin exchange is, in turn, examined in the context of CIDEP radical pair mechanism, chemical reaction distances, and intersystem crossing of radical pairs.

In each of the following sections, we will consider one sort of exchange, look at its relevance to the other exchange interactions, and summarize some of its manifestations. Because many manifestations of "exchange" are found in magnetic resonance and because we present some previously unpublished experiments of our own, an emphasis is placed on exchange as it relates to time-resolved esr (TRESR) and chemically induced dynamic electron polarization (CIDEP). Clearly, other approaches could have been taken to make the same point. Where complex mathematics are involved in derivations, usually only the meaningful results are given, in hope of presenting the reader with a useful physical picture. The literature cited is meant to be representative, rather than exhaustive.

TRESR allows the observation of radicals whose lifetimes are too short to be seen by steady state techniques. Moreover, radicals created by flash photolysis in the esr cavity very often exhibit non-Boltzmann spin distributions (i.e. spin polarization) called CIDEP. Several recent reviews have very nicely described the mechanisms of CIDEP polarizations [1], so we will give only a brief explanation here. The two most important polarization mechanisms are the triplet mechanism (TM) and the radical pair mechanism (RPM). They are somewhat complementary in that they 
yield information on the radical formation steps and on the subsequent radical pair dynamics, respectively. Fortunately, also, they are readily distinguishable on inspection of spectra.

Triplet mechanism results in the actual overpopulation of $\alpha$ or $\beta$ electron spin states. The hyperfine lines are proportionally overpopulated so that the spectrum is affected only in intensity and sign. TM polarization is mainly observed in very fast abstraction and cleavage reactions of excited triplet carbonyl compounds and azaaromatics. It comes about because of selection rules in the $S_{1} \rightarrow T_{n}$ intersystem crossing step that causes overpopulation of one sublevel with particular symmetry (Figure 1a) $[2]$.<smiles>[3H]C([3H])=O</smiles>
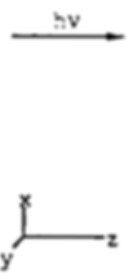
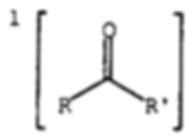
${ }^{2}\left(\pi, \pi^{*}\right)$

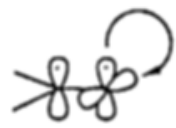

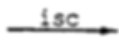

$\frac{\text { electron }}{\text { rotation" }}$

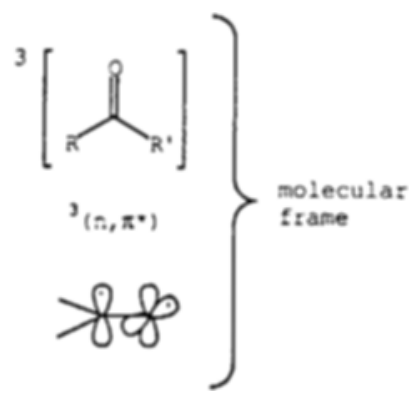

Figure 1a. A schematic representation of the triplet mechanism. (a) Excitation of a ketone into a ${ }^{1} \pi, \pi^{*}$ state is followed by spin-orbit mediated intersystem crossing. The "rotation" of the electron from a $\mathrm{p}_{x}$ to a $\mathrm{p}_{y}$ orbital causes angular momentum in the $z$ direction, thus populating the $T_{z}$ level preferentially.

When the mechanism for intersystem crossing is spin-orbit coupling, the selection rule for intersystem crossing (ISC) from the excited singlet to a set of triplet sublevels can be summarized by the condition that

$$
\Gamma(S) \otimes \Gamma(R n) \otimes \Gamma(T) \text { contains } A_{1 g},
$$

where $\Gamma$ is the symmetry of a state or operator, $R_{n}$ is the rotation operator for $\mathrm{x}, \mathrm{y}$, or $\mathrm{z}$, and $\mathrm{T}_{n}$ is the triplet sublevel whose symmetry corresponds to the rotation operator. Generally, this condition holds for only one of the sublevels, which is then overpopulated. The selection rule can be rationalized for carbonyls by noting that ISC from $\pi, \pi^{*}$ to $n, \pi^{*}$ states or the reverse involves a "rotation" of the electron density in the $x, y$ plane, causing a resultant electron angular momentum in the $\mathrm{z}$ direction (Figure 


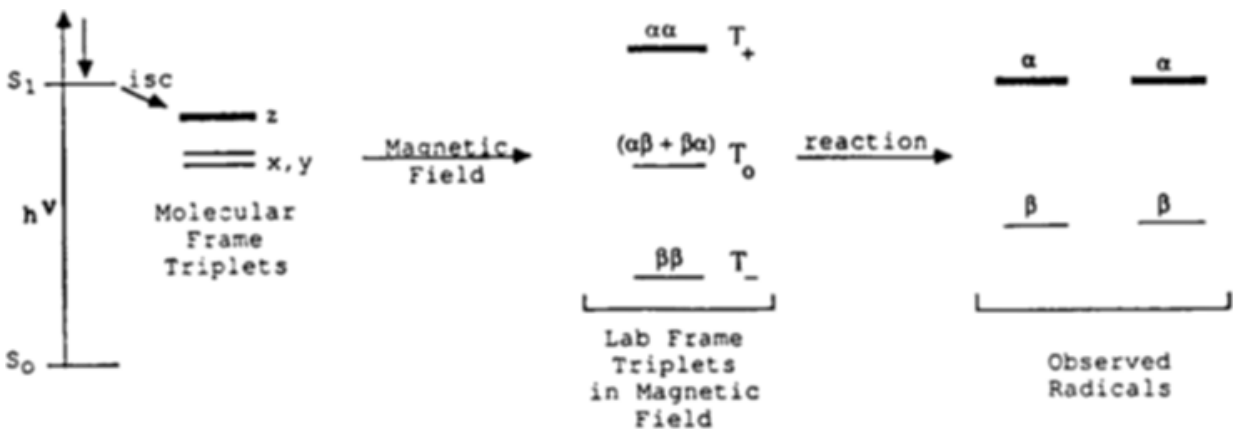

Figure 1b. A schematic representation of the triplet mechanism. (b) The intersystem crossing step into the molecular frame triplet is shown in the first energy diagram with the overpopulated $T_{z}$ level represented by the thick bar. The triplet levels then interact with the magnetic field. In the laboratory frame, much of the polarization is transferred to the $\mathrm{T}_{+}$level. The molecule undergoes a fast reaction, yielding radicals which are polarized with excess $\alpha$ spin.

1b). Spin orbit coupling in ketones fills the $T_{z}$ level preferentially, as predicted by this explanation.

Of course, polarization generated by intramolecular selection rules is in the molecular frame only. By measuring the polarization in the magnetic field of an esr spectrometer, the molecular frame triplets are forced to interact with the laboratory frame field to yield the set of laboratory frame triplets $\mathrm{T}_{+}, \mathrm{T}_{o}$, and $\mathrm{T}_{-}$from the molecular frame triplets $\mathrm{T}_{x}, \mathrm{~T}_{y}$, and $T_{z}$. Fortunately (for those interested in CIDEP experiments!) the highest triplet sublevel in the molecular frame contributes the most to $\mathrm{T}_{+}$ when all orientations are averaged, and the lowest molecular frame triplet contributes the most to $T_{-}$. Thus, the molecular frame polarization can be largely maintained in the laboratory frame. Clearly, overpopulation of the $\mathrm{T}_{o}$ level does not lead to polarization.

The electron spin moments of the triplet can only react to the magnetic field at a rate given by the Zeeman frequency $\omega_{z}=\mathrm{g} \beta \mathrm{H} / \hbar$. In order for the polarization to be efficiently maintained $\omega_{z}$ must not be much faster than the rate at which the electrons respond to each other, $\omega_{Z F S}$ $=\mathrm{D} / \mathrm{h}$, nor may it be much slower than the rotational correlation time $\tau_{c}$.

The relaxation time of spin polarization in triplets at room temperature, often being $10^{-8}$ to $10^{-10} \mathrm{~s}$, is too short to observe polarization in triplets directly. In the example where the highest triplet sublevel is 
overpopulated, as in aromatic carbonyls [3] excess $\alpha$ spin is found in the triplet. If radical-forming reactions occur before the triplet spins thermalize (i.e., return to Boltzmann distribution), then the radicals are formed with excess $\alpha$ spin. The relaxation times of the new doublets are typically much longer, of the order of a microsecond, which is sufficient for observation.

Figure 2 shows a spectrum obtained on the photolysis of benzil dimethylmonoketal, which exhibits TM polarization. Only the dimethoxy benzyl radical is observed during the time window used for this particular spectrum (ca. $1-2 \mu$ s after the flash); the benzoyl radical has a comparatively fast relaxation time, loses its spin polarization quickly, and is not detected in this time window. Spectra taken at earlier times (which are uncertainty broadened) show both radicals in emission.
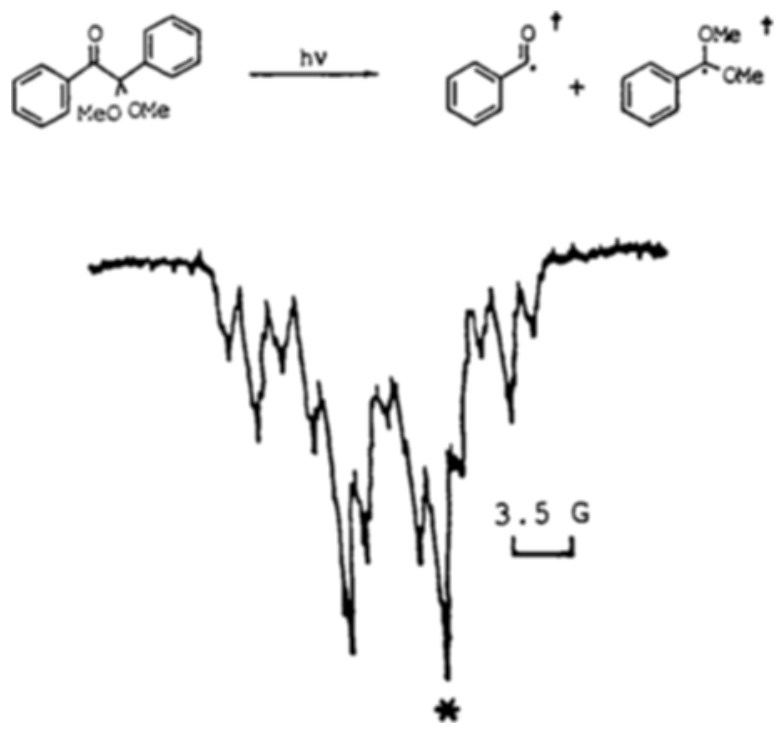

Figure 2. The TRESR spectrum obtained on photolysis of benzil dimethylmonoketal. It is all in emission and each line is in proportion to its degeneracy due to TM polarization. The peak marked with a star contains all of the intensity due to the benzil radical.

In contrast, the radical pair mechanism (RPM), or $\mathrm{ST}_{0}$ mechanism has nothing to with a molecular intersystem crossing step and may arise from either singlet or triplet precursors. The expected observation in the absence of competing relaxation mechanisms or net polarization is 
equal and opposite polarization in hyperfine lines of the same absolute magnitude (i.e. $\pm 1, \pm 2$, etc.) and zero polarization of the center line, if any (assuming $g_{1} \approx g_{2}$ ). No net polarization is observed, in that the total $\alpha$ and $\beta$ spins are in Boltzmann distribution. This is a key difference between RPM and TM when exchange effects are considered, as discussed below.

RPM polarization comes about because of spin exchange between re-encountering radical pairs and will be discussed in more detail in section IIIA. Figure 3 shows the TRESR spectrum obtained on photolysis of acetone in isopropanol, which shows RPM polarization superimposed on a small amount of net absorptive polarization. Often spectra show both RPM and TM, so the appearance of the spectrum is dependent on the relative contributions of those mechanisms.

Other polarization mechanisms, notably $\mathrm{S} / \mathrm{T}_{-}$mixing [4] and passive CIDEP [5] can be important under special conditions. The former mechanism is observed particularly when hyperfine coupling constants are very large, such as with the hydrogen atom $[6,7]$, phosphoranyl radicals [8], or when the solvent viscosity is very high [9]. The latter mechanism becomes most important when radical recombination rates approach the radical electronic $T_{1}\left(c a .10^{6} \mathrm{~s}^{-1}\right)[5]$.

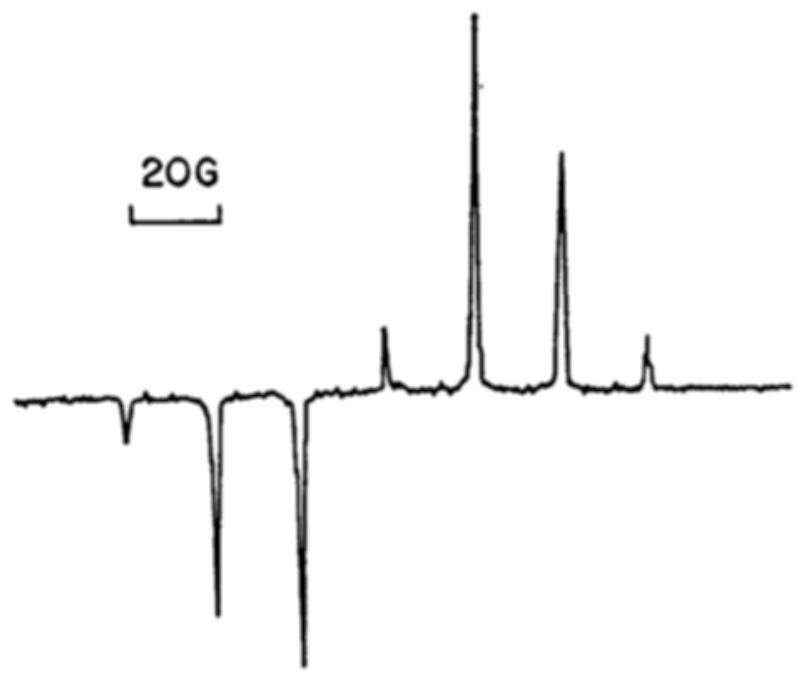

Figure 3. The TRESR spectrum obtained on photolysis of solutions of acetone in isopropanol. RPM polarization is superimposed on a net absorptive polarization. 


\section{ELECTRONIC AND SPIN EXCHANGE}

\section{A. The exchange interaction}

In the worlds of quantum mechanics and magnetic resonance, there are exchange integrals, and then there are exchange integrals. The exchange integral found in all the quantum mechanics textbooks is traditionally labeled $\mathrm{K}$ and has the form used in the introduction, i.e.,

$$
K_{\text {quantum }}=<\phi_{a}(1) \phi_{b}(2)\left|e^{2} / r_{12}\right| \phi_{b}(1) \phi_{a}(2)>
$$

Much of the magnetic resonance literature is full of references to something termed $\mathrm{J}$, the "exchange integral", which represents the energy difference between the $S$ and $T_{0}$ states of a pair of interacting electron spins. As often as not, explicit mention is made that $\mathrm{J}_{\text {mag res }}=-2 \mathrm{~K}_{\text {quantum }}$.

The reality is that $J_{m a g}$ res $=-2 \mathrm{~K}$ in only one limit: when there is zero net overlap between the localized orbitals containing the unpaired spins and when those orbitals are degenerate. Such are, of course, the limits in which Hund's rule applies.

The confusion is made only worse by the fact that $\mathrm{J}$ is also traditionally used in the quantum mechanics literature as the Coulomb integral,

$$
J_{\text {quantum }}=<\phi_{a}(1) \phi_{b}(2)\left|e^{2} / r_{12}\right| \phi_{a}(1) \phi_{b}(2)>
$$

Despite their similar form, $\mathrm{J}_{\text {quantum }}$ and $\mathrm{K}$ are totally different quantities, the former with classical analog, and the latter without. As a functional example, $\mathrm{J}_{\text {quantum }}$ and $\mathrm{K}$ are the matrix elements important in dipole-dipole and exchange mediated energy transfer, respectively. The implications of $\mathrm{J}_{\text {quantum }}$ and $\mathrm{K}$ for spin polarization in energy transfer are examined in section IIIF.

$\mathrm{J}_{m a g}$ res is more realistically viewed as an empirical parameter, which can just as well be called the triplet-singlet energy gap, $\Delta \mathrm{E}_{T_{o}} s$. One realizes that $\mathrm{J}_{\text {mag res }}$ is not generally $-2 \mathrm{~K}$ because $\mathrm{J}_{\text {mag res }}$ almost always favors a singlet configuration, whereas $\mathrm{K}$ always favors a triplet. This will be seen below to be due to overlap considerations. Even the biradical literature is filled with two non-interacting sets of experiments, one of which uses the "proper" $\mathrm{J}$ and $\mathrm{K}$ terminology, and one (mainly concerned with magnetic resonance effects-see section IIIC) that uses the more ambiguous terminology.

In an attempt to deal explicitly with the differences between $\mathrm{J}_{\text {mag res }}$ and $K_{\text {quantum }}$, we briefly review here some work from the biradical literature which expresses $\Delta \mathrm{E}_{T_{o} S}$ in terms of $\mathrm{K}_{\text {quantum }}$. Salem and Rowland pointed out two ways of describing a biradical [10]. The simpler is to 
assign each unpaired spin to a localized orbital, and then to allow interaction, particularly exchange, $\mathrm{K}$, and overlap, $\mathrm{S}$ (Coulomb interactions do not distinguish singlet and triplet states.). It is this model on which most of the interpretation of the spin interaction between nitroxide biradicals is based [11]. The localized biradical picture is lost in two cases: if the overlap is too large, bond formation occurs, and if the energy difference between the two localized orbitals is too large, electron pairing will occur in the lower orbital. On the other hand, if the $\mathrm{S}$ and $\mathrm{K}$ are essentially zero, the molecule behaves as if it were only a pair of doublets.

The second model is more commonly used in energy calculations and considers two nearly degenerate molecular orbitals. Two different papers came out almost simultaneously which gave explicit functions for $\Delta \mathrm{E}_{T S}$ $[12,13]$. We will use the notation of Doubleday, et al. [12] simply because it is simpler to visualize.

We begin with two singly occupied localized orbitals with wave functions $\chi_{1}$ and $\chi_{2}$. Next the two radicals are brought together in the orientation of interest, and $\chi_{1}$ and $\chi_{2}$ are allowed to mix, giving orthogonal orbitals $\phi_{a}$ and $\phi_{b}$. (If other orbitals are available of the proper symmetry, they will also mix into $\phi_{a}$ and $\phi_{b}$.) Now, if the overlap integral $S_{12}$ between $\chi_{1}$ and $\chi_{2}$ is 0 , then they remain as unchanged orthogonal nonbonding orbitals, but if $S_{12} \neq 0$, then $\phi_{a}$ and $\phi_{b}$ represent the antibonding and bonding combinations, respectively. The exchange integral $K_{a b}$ over $\phi_{a}$ and $\phi_{b}$ will always favor single occupancy in each orbital (i.e. a triplet state), but the actual configuration is determined by the magnitude of $\mathrm{K}^{\prime}$ compared to $\Delta \mathrm{E}_{a b}$ (Figure 4). $\Delta \mathrm{E}_{T S}\left(\mathrm{~J}_{m a g \text { res }}\right.$ ) itself, then depends on

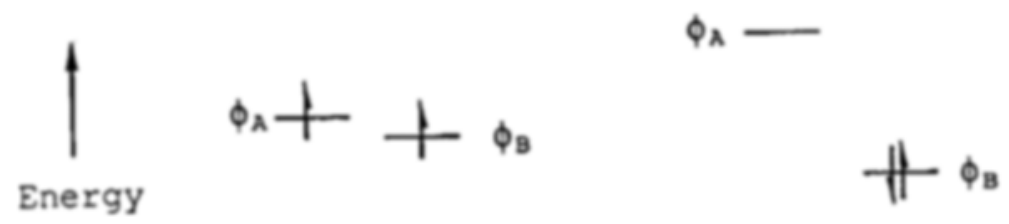

Figure 4. If the energies of $\phi_{A}$ and $\phi_{B}$ are very close, the exchange integral will overcome the energy difference and the biradical will be a triplet. If the energy difference is large, a singlet will be formed.

the overlap of the localized atomic orbitals and their relative energies $[12,14]$ :

$$
\Delta E_{S T}=J_{m a g \text { res }}=-2 K^{\prime}-K_{a b}+\left[\left(\Delta E_{a b}\right)^{2}+K_{a b}^{2}\right]^{1 / 2}
$$


$\mathrm{K}^{\prime}$ is the exchange integral over $2^{-1 / 2}\left(\phi_{a}+\phi_{b}\right)$ and $2^{-1 / 2}\left(\phi_{a}-\phi_{b}\right), \mathrm{K}_{a b}$ is defined above, and $\Delta \mathrm{E}_{a b}$ is the energy difference between the antibonding and bonding molecular orbitals. If only $\chi_{1}$ and $\chi_{2}$ contribute to $\phi_{a}$ and $\phi_{b}$, then $K^{\prime}=K_{12}$. The expression simplifies even further if $S_{12}=0$, since $\mathrm{K}_{12}=\mathrm{K}_{\mathrm{ab}}$. If $\mathrm{S}_{12}$ is small, as is normal for biradicals or radical pairs, then

$$
\Delta E_{a b} \approx \Delta E_{12}+c S_{12}
$$

where $\Delta E_{12}$ is the energy difference between $\chi_{1}$ and $\chi_{2}$ and $c$ is a proportionality constant. From this expression, it can be shown that when $c^{2} \mathrm{~S}_{12}^{2}<<\mathrm{K}_{a b}^{2}, \Delta \mathrm{E}_{T S}$ is approximately proportional to the square of $\mathrm{S}_{12}$ $[14,15]$.

Equation 4 shows that $\mathrm{J}_{\text {mag res }}=-2 \mathrm{~K}$ only when $\mathrm{S}_{12}=0$ and when $\Delta \mathrm{E}_{a b}=0$. Realistically, $\Delta \mathrm{E}_{a b}$ is small in most measurements of $\mathrm{J}$. In all other cases the so-called exchange interaction of magnetic resonance actually has a number of terms. It should also be pointed out that different techniques for the measurement of $\mathrm{J}$ will lead to different answers. We will try to point out the origins of this when it comes up. Due to its overwhelming usage, we will use $\mathrm{J}$ many times in the remainder of this review, when $\Delta \mathrm{E}_{T_{o} S}$ is really what is meant. Where possible, we will use words other than "the exchange interaction" to describe it. Table I summarized the important equations used here and below.

\section{TABLE I}

\begin{tabular}{|c|c|c|}
\hline Symbol & Name & Explanation \\
\hline $\mathrm{K}$ & exchange & $\begin{array}{l}<\phi_{a}(1) \phi_{b}(2)\left|e^{2} / r_{12}\right| \phi_{b}(1) \phi_{a}(2)> \\
\text { always favors triplet configuration }\end{array}$ \\
\hline $\mathbf{J}_{\text {quantum }}$ & coulomb & $\begin{array}{l}\left.<\phi_{a}(1) \phi_{b}(2)\left|e^{2} / r_{12}\right| \phi_{a}(1) \phi_{b}(2)\right\rangle \\
\text { does not differentiate between singlets } \\
\text { and triplets }\end{array}$ \\
\hline $\mathbf{S}$ & overlap & $\begin{array}{l}<\phi_{a}(1)\left|\phi_{b}(1)\right\rangle \\
\text { favors singlet by making bonding and } \\
\text { antibonding combinations }\end{array}$ \\
\hline $\mathrm{J}_{\text {mag res }}$ & "exchange" & $\begin{array}{l}\mathrm{E}_{T o}-\mathrm{E}_{s} \text { which equals }-2 \mathrm{~K} \text { at the limit } \\
\text { of } \mathrm{S}=0 \text { and degenerate orbitals }\end{array}$ \\
\hline
\end{tabular}




\section{B. Spin exchange}

Key to many of the phenomena that will be discussed is the very well known phenomenon of Heisenberg spin exchange [15]. The most important type of spin exchange ("strong exchange") occurs in solution when two paramagnetic particles of opposite spin approach each other to within a van der Waals radius or so and swap total spin orientation in what is sometimes known as a flip-flop transition. A simple illustration is given in Figure 5.

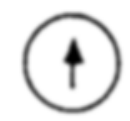

A

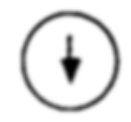

B

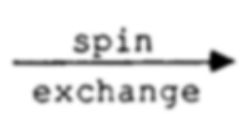

A

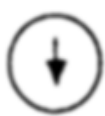

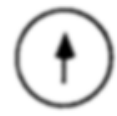

B

Figure 5. Spin exchange between particles of opposite spin.

For radicals in solution, the energy of spin exchange is well described by the Hamiltonian [16]

$$
H_{e x}=\hbar \cdot J_{A B}(r) \cdot \mathbf{S}_{A} \cdot \mathbf{S}_{B}
$$

where $\mathrm{J}_{A B}(\mathrm{r})$ is the singlet-triplet splitting of the "radical pair". $\mathbf{S}_{A}$ and $S_{B}$ are the spin operators of the two particles. This energy, typically $10^{11}$ to $10^{12} \mathrm{~Hz}$ for molecules is generally smaller than the thermal energy of motion ( $\mathrm{kT} / \mathrm{h}=c a \cdot 10^{13} \mathrm{~Hz}$ at room temperature) and thus does not affect diffusional motion.

The most common experimental manifestation of spin exchange is the broadening of individual hyperfine lines of steady state esr spectra [15]. Several other effects, including RPM polarization, are also related to spin exchange, so we review and summarize its cause, vocabulary, and benchmark values here, for the most part restricting ourselves to the case of collisions between two spin $1 / 2$ particles.

Perhaps the most important parameter of spin exchange is its rate, which can be expressed as

$$
k_{e x}=P_{e x} \cdot k_{d i f f}
$$

$P_{e x}$ is the probability that a collision will result in spin exchange and $\mathrm{k}_{\text {dif } f}$ is the rate of collisions. A fudge factor can be added to account for steric interactions which blocks effective collision, yielding $k_{e x}=f \cdot P_{e x}$. $\mathbf{k}_{\text {diff }}$. 
Consider what happens when two spin $1 / 2$ particles $A$ and $B$ are brought together. We will assume that they are identical, that they have no significant hyperfine coupling, and that they have opposite spin. If the encounter is a very brief one, with a low effective $J$, then we expect that $\mathrm{P}_{\text {ex }}$ will be small. If a "strong" collision occurs, it is expected that the spins will totally randomize, leading to a maximum $P_{e x}$ of 0.5 . These expectations are borne out in calculations that are summarized below.

Particles with opposite spins can, of course, couple into either an S or $T_{0}$ state. The initial electron spin-only wave function can then be written as

$$
\Psi(t=0)=C_{S}(0) \Psi_{S}+C_{T}(0) \Psi_{T_{\circ}}
$$

where $\mathrm{C}_{S}(\mathrm{t})$ and $\mathrm{C}_{T}(\mathrm{t})$ are time dependent coefficients of the singlet and triplet wave functions

$$
\begin{aligned}
& \Psi_{S}=2^{-1 / 2}\left(\left|\alpha_{A} \beta_{B}>-\right| \beta_{A} \alpha_{B}>\right) \\
& \Psi_{T_{0}}=2^{-1 / 2}\left(\left|\alpha_{A} \beta_{B}>+\right| \beta_{A} \alpha_{B}>\right)
\end{aligned}
$$

Because the energy of the singlet and triplet wave functions differ by $\mathrm{J}, \mathrm{C}_{S}$ and $\mathrm{C}_{T}$ are out of phase with each other with respect to time. In order to calculate $\mathrm{C}_{S}(\mathrm{t})$ and $\mathrm{C}_{T}(\mathrm{t})$, we will use the "contact exchange model" in which $\mathrm{J}$, the singlet triplet splitting, is zero unless the radicals are in closest contact. According to this model, the exchange interaction is turned on when the two particles collide and goes directly to zero when the particles diffuse apart at all. Moreover, unless the orbitals in question are perfectly symmetric (i.e., s orbitals), there is an angular dependence on $\mathrm{J}$ due to the change in overlap integral S. Thus $J(r)$ is actually anisotropic with respect to the mutual orientation of the orbitals. However, most treatments, including this one, implicity or explicitly assume that the rotational correlation time is sufficiently fast to use only an average $J$ [15]. Calculations that map out $\mathrm{J}$ through the orientation space of a pair of radicals are not simple [21], but have been carried out simple systems, notably $\mathrm{H}$ plus $\mathrm{CH}_{3}[22]$.

Applying the time dependent Schrödinger equation i $\hbar \delta \Psi / \delta \mathrm{t}=\mathrm{H}_{e x}$ $\Psi$, one obtains the expressions for $\mathrm{C}_{S}(\mathrm{t})$ and $\mathrm{C}_{T}(\mathrm{t})$ :

$$
\begin{gathered}
C_{S}(t)=C_{s}(0) \exp (i J t / 2)=\cos (J t / 2) C_{s}(0)-i \sin (J t / 2) C_{S}(0) \\
C_{T}(t)=C_{T}(0) \exp (-i J t / 2)=\cos (J t / 2) C_{T}(0)-i \sin (J t / 2) C_{T}(0)
\end{gathered}
$$


If $\Psi(0)$ is taken to be $\mid \alpha_{A} \beta_{B}>$ and one asks the probability of going $\Psi(\mathrm{t})$ $=\left|\beta_{A} \alpha_{B}\right\rangle$ (i.e., the probability of spin exchange after time $t$ ), the result is [15].

$$
P=\sin ^{2}(J t / 2)
$$

This simple equation yields the basis for the distinction between "strong exchange" and "weak exchange". If $\mathrm{Jt} \ll 1$, then the transition probability is very small, since $\sin ^{2}(\mathrm{Jt} / 2)$ goes to zero; this is weak exchange. If $\mathrm{Jt}>>1$ (strong exchange), then the spins are completely randomized as integration of $\mathrm{P}$ over $\mathrm{t}$ or $\mathrm{J}$ gives $\mathrm{P}=0.5$.

Another approach to calculating exchange rates involves density matrix methods. Each particle is assigned a $2 \times 2$ one-particle spin density matrix. The interaction is written by making a two-particle spin density matrix applying the stochastic Liouville equation. Moreover, a more realistic approximation of $\mathrm{J}$ is used. It allows a distance dependence on $\mathrm{J}$, which is usually assumed to be exponential, giving

$$
J(r)=J_{o} e^{-\kappa r}
$$

Estimates of $\mathrm{J}_{o}$ are dependent on the decay steepness parameter $\kappa$ [17], but reasonable ones are in the range of $10^{10}$ to $10^{13} \mathrm{~Hz}$, depending on whether atomic or molecular systems are considered $[15,17,18]$. Both experimental and theoretical work indicates that $4<\kappa \mathrm{r}_{0}<10$, where $\mathrm{r}_{0}$ is the distance of closest approach for the radical pair $[4 \mathrm{~b}, 19,20]$.

The basic result of the density matrix method is $[15,23]$

$$
P=1 / 2 \cdot\left[J^{2} t_{c}^{2} /\left(1+J^{2} t_{c}^{2}\right)\right]
$$

where $t_{c}$ is the average "collision" time. The weak and strong exchange limits are very similar for this formulation of $\mathrm{P}$ as for Eq. (11). In the strong exchange limit, $\mathrm{Jt}_{c}>>1$ and $\mathrm{P}=1 / 2$. Again, $\mathrm{P}$ shows no dependence on $\mathrm{J}$ in the strong limit. In the weak exchange limit $\mathrm{P}$ is proportional to $\mathrm{J}^{2} \mathrm{t}_{\mathrm{c}}^{2}$ in both formulations as well.

The total rate of exchange is still a matter of some confusion in the literature. Some authors $[15,17,24]$ state that in the strong exchange limit

$$
k_{e x}=1 / 2 k_{d i f f}
$$

while others $[19,25]$ stick to the apparent result that

$$
k_{e x}=1 / 4 k_{d i f f}
$$

which one obtains by considering only $\mathrm{S}$ and $\mathrm{T}_{0}$ collisions. 
The confusion arises because it is not obvious whether or not to include $\mathrm{T}_{ \pm}$radical pairs, although one author claims that $\mathrm{k}_{e x, \max }=1 / 2$ $\mathrm{k}_{\text {diff }}$ because every strong $\mathrm{S}$ or $\mathrm{T}_{0}$ collision leads to exchange [24]. A more reasonable interpretation comes from Syage [17,26], who notes in his treatment (which includes all possible spin states) that exchange occurs between radicals in $T_{ \pm}$pairs, even though it is unmeasurable. Moreover, an exchange event between $\mathrm{S}$ or $\mathrm{T}_{0}$ pairs contributes twice the relaxation as an ordinary spin-lattice event, thus "covering" for the unmeasured exchange events. The rate in $14 \mathrm{~b}$ is also sometimes known as "spectral exchange".

The experimental results $[27,28]$ seem to indicate that $1 / 2 \mathrm{k}_{\text {diff }}$ is correct. For relatively unhindered stable nitroxides, the measured rates are commonly $75-105 \%$ of $1 / 2 \mathrm{k}_{\text {diff } f}$ [15], the latter being calculated with

$$
k_{d i f f}=8 R T / 3000 \eta
$$

Spin exchange is most commonly detected and its rate is most commonly quantified by the measurement of the peak-to-peak line widths of steady state esr spectra. In the slow exchange rate limit (i.e., where the lines are not broadened to the point of merging to a single line), the rate constant for exchange $\left(k_{e}\right)$ is found with the following Eq. (15)

$$
k_{e}=[R \cdot] \cdot \Delta \omega_{1 / 2} /(1-\phi)
$$

where $[R \cdot]$ is the concentration of the exchanging radical, $\Delta \omega_{1 / 2}$ is the observed width of the line in $\mathrm{Hz}$ less the inherent linewidth, and $\phi$ is the statistical weight of the particular hyperfine line. This equation may be used to measure either self exchange or exchange between a given radical and another whose spectrum is being monitored. As was pointed out over twenty years ago [29], degenerate electron exchange reactions

$$
A .^{-}+A \leftrightarrow A+A .^{-}
$$

also lead to essentially the same broadening effects as spin exchange if the rates are similar. Degenerate electron exchange, however, does have unique effects on the observed electronic relaxation [30].

\section{EXPERIMENTAL EXCHANGE PHENOMENA}

\section{A. Radical pair mechanism and spin exchange}

The production of RPM requires spin exchange. However, as we have just seen, "strong" spin exchange serves only to randomize spins. It turns 
out that RPM is best produced on collisions where $\mathrm{Jt}_{c} \approx 1$. In fact, there is always a competition between spin exchange and RPM polarization, with the former serving to attenuate the latter. This section gives some of the qualitative details for the production of RPM polarization in order to explore the relationships between the two processes. We will see that fairly weak exchange serves to help polarize previously correlated pairs, while strong exchange depolarizes the spin pool.

Since RPM is commonly observed in solutions of reactive radicals, we know that it competes favorably with strong spin exchange and with even diffusion controlled reactions. The generally accepted theory of RPM polarization requires that a radical pair undergo a two step process to produce polarization: hyperfine mixing of the $S$ and $T_{o}$ states followed by weak spin exchange. Collisions leading to $T_{ \pm}$pairs are assumed not to contribute to the polarization. Following Adrian's logic [1b,31], since hyperfine couplings are on the order of $10^{8} \mathrm{~Hz}$, it must take at least the order of $10^{-8} \mathrm{~s}$ for mixing to take place (by the uncertainty relationship). This is far longer than the typical encounter time in non-viscous solvents, so it was proposed [32] that radical pairs which encounter each other and then re-encounter some time later without having lost their spin correlation are responsible for RPM.

To calculate the RPM polarization, the time dependent wave function of a pair of colliding radical spins is again written as in section IIB.

$$
\Psi(t)=C_{S}(t) \Psi_{S}+C_{T}(t) \Psi_{T_{o}}
$$

The off diagonal part of the Hamiltonian which will allow mixing between the singlet and triplet states is [33]

$$
H^{\prime}=Q+H_{e x}=\left(g_{1}-g_{2}\right) \beta_{e} B_{o} / \hbar+(1 / 2)\left(\Sigma A_{j} \mathbf{I}_{j}-\Sigma A_{k} \mathbf{I}_{k}\right)+J(r) \mathbf{S}_{1} \cdot \mathbf{S}_{2}
$$

The parameter $Q$ is simply the difference in resonant frequency for the two radicals. For almost all organic radicals, $Q$ is dominated by the hyperfine term, so we refer to $\mathrm{Q}$ mixing as hyperfine mixing here. The RPM polarization of a given hyperfine line is given by [31]

$$
\operatorname{Pol}(t)=C_{T_{0}}{ }^{*}(t) C_{S}(t)+C_{S}{ }^{*}(t) C_{T_{0}}(t)
$$

where the asterisk denote a complex conjugate, so all that must be done is calculate the coefficients $\mathrm{C}_{T_{0}}(\mathrm{t})$ and $\mathrm{C}_{S}(\mathrm{t})$.

When radicals encounter each other in solution, there are three possible initial conditions $[35,36]$. The first two are that the radicals are a geminate pair produced via either a singlet or triplet state reaction. In 
the former case, $\mathrm{C}_{S}(0)=1$ and $\mathrm{C}_{T}(0)=0$. In the latter, the values are reversed. The third case is when the radicals encounter each other randomly, which is called $\mathrm{F}$-pairing, leading to equal numbers of $\mathrm{S}$ and $\mathrm{T}_{0}$ pairs. It is the depletion of singlet pairs due to reaction that is required for F-pair polarization, leading to an overall triplet behavior. We will see below that equal and opposite polarization is generated from (nonreacting) singlet and triplet pairs. This is why RPM is not observed for solutions of persistent radicals.

In order to calculate the RPM polarization, we will examine, in sequence, the effects of hyperfine mixing and spin exchange on the wave function. Hyperfine coupling constants are of the order of $10^{8} \mathrm{~Hz}$, whereas $\mathrm{J}_{o}$ is of the order of $10^{12} \mathrm{~Hz}$ during collision, so hyperfine mixing must occur when the radicals have diffused apart several molecular diameters. We simplify this treatment by again using the contact exchange model for $\mathrm{J}$, instead of the more realistic Eq. (12). Physically, the following treatment can be visualized with the aid of Figure 6. After the pair is born (as a triplet, for instance), it begins to diffuse apart. As a result, the exchange interaction drops to zero and hyperfine interactions can mix the $\mathrm{T}_{o}$ and $\mathrm{S}$ states. Some of the diffusing pairs will reencounter. When they do, we assume that $J>Q$, so that we may treat it independently. This scheme, which is actually a fairly crude approximation, justifies our stepwise approach to the calculation. The reencounter dynamics are stuck on at the end.
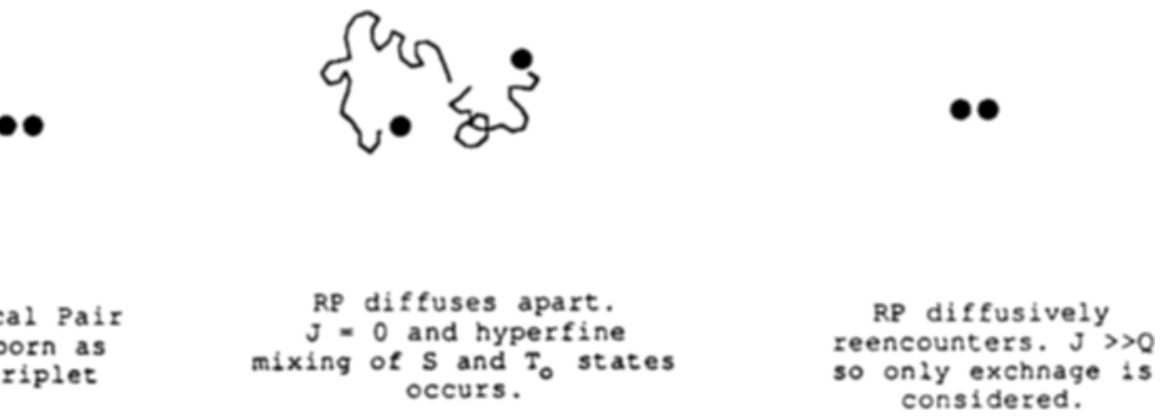

Figure 6. A schematic representation of the model used to calculate RPM polarization.

After hyperfine mixing has been considered, the time dependent coefficients of the wave function are $[31,35]$ 


$$
\begin{aligned}
& C_{S}(t)=\cos (Q t) C_{S}(0)-i \sin (Q t) C_{T}(0) \\
& C_{T}(t)=\cos (Q t) C_{T}(0)-i \sin (Q t) C_{S}(0)
\end{aligned}
$$

We check now to see if any polarization has come about due only to hyperfine mixing using Eq. (20). Applying the coefficients from Eq. (21) to Eq. (20), the polarization is found to be

$$
\operatorname{Pol}(t)=C_{S}(0) C_{T}{ }^{*}(0)+C_{S}{ }^{*}(0) C_{T}(0)=0
$$

Equation 22 shows that no polarization is observed, since one of the constants $\mathrm{C}_{i}(0)=0$.

If, however, we allow the radical pair to diffuse back together and undergo exchange interactions for a period of time $\tau$, further mixing occurs. We use a treatment similar to section IIB, only substituting the current $\mathrm{C}_{i}(\mathrm{t})$ values for $\mathrm{C}_{i}(0)$ in Eq. (10). After hyperfine mixing, and allowing spin exchange, the coefficients are

$$
\begin{gathered}
C_{S}(t, \tau)=\left[\cos (Q t) C_{S}(0)-i \sin (Q t) C_{T}(0)\right] e^{-i J \tau} \\
C_{T}(t, \tau)=\left[\cos (Q t) C_{T}(0)-i \sin (Q t) C_{S}(0)\right] e^{i J \tau}
\end{gathered}
$$

Calculation of the polarization leads to several terms. Some of them depend on $\mathrm{C}_{S}(0) \mathrm{C}_{T}{ }^{*}(0)$ or its complex conjugate, which is still equal to 0 . However, the following term also falls out:

$$
\operatorname{Pol}(t, \tau)=\left[C_{S}(0) C_{S}{ }^{*}(0)-C_{T}(0) C_{T}{ }^{*}(0)\right] \sin (2 Q t) \sin (2 J \tau)
$$

This polarization term is non-zero (barring problems with the arguments of the sin terms) because regardless of whether $\mathrm{C}_{S}(0)$ or $\mathrm{C}_{T}(0)$ is zero, the other is 1 .

Equation (24) is also very helpful in picturing the signs of the polarization. It shows that radical pairs born as singlet and triplet states will have opposite polarization. F-pairs of stable radicals will be half singlet and half triplet, so no net polarization is observed for them. Moreover, $Q$ is negative for lines on the low field half of the spectrum and positive on the upper field half. From this comes the E/A or A/E pattern. It can also be seen that strong exchange $(\mathrm{J} \tau>>1)$ will randomize the incipient polarization, as one would expect, while very weak exchange $(\mathrm{J} \tau<<1)$ leads to very little polarization. Clearly the optimum is a "moderate" exchange interaction, where $\mathrm{J} \tau=\pi / 4 \approx 1$.

Now we must consider the reencounter dynamics. There is a distribution of times over which the radical pairs diffuse back together after 
the hyperfine interaction and before exchange. Of course, many never reencounter. Concomitant with the variation of return times are different degrees of singlet triplet mixing. After integrating over this distribution, one obtains [31]

$$
P o l=.85 \operatorname{sign}(Q J)\left(Q / t_{d}\right)^{1 / 2}\left[C_{S}(0) C_{S}{ }^{*}(0)-C_{T}(0) C_{T}{ }^{*}(0)\right] \sin (2 J \tau)
$$

where $t_{d}$ is the mean time between diffusive displacements (ca. $10^{-11}$ $10^{-12} \mathrm{~s}$ ) and is inversely proportional to the diffusion constant. There are conditions in which polarization is proportional to $\mathrm{Q}$, rather than $\mathrm{Q}^{1 / 2}$, but we will not discuss them here $[1,17]$.

The RPM polarization is quantifiable using numerical solutions of the stochastic Liouville equations that describe the spin behavior $[33,34]$. Pedersen and Freed explicitly assumed that $\mathrm{J}$ was only a function of $\mathrm{r}_{a b}$ (i.e., that rotational dependences are averaged out). They found that the contact exchange model was inadequate and that the exponential decay model (Eq. (12)) was more satisfactory.

Polarizations calculated by the Pedersen and Freed formulae are consistently too small by a factor of $2-4$ compared to measured polarizations [1c]. Various authors have extended this treatment [34] or modified it by solving analytically the equations by making limiting assumptions [36]. Other extensions, which will not be discussed here, include the explicit accounting for anisotropy [37] and mixing with the $\mathrm{T}_{+}$and $\mathrm{T}_{-}$states [38].

No net polarization is created by RPM; only a redistribution of $\alpha$ and $\beta$ spins occurs. Thus it is quite reasonable to expect that spin exchange between random radical pairs should lead to attenuation of RPM. Such is the case. Spin exchange between radical pairs in different hyperfine lines does not return the spin populations of those two lines to the Boltzmann distribution, but rather to the average polarization of the two hyperfine lines involved [39]. Many experiments in which radical anions were the observed species seemed to show strong TM polarization and no RPM, which is uncommon in other systems [30]. As mentioned above, degenerate electron exchange can have the same effects as spin exchange; it was later recognized that electron exchange with radical anions was so rapid as to completely wipe out any RPM that might have developed [40]!

The balance between polarization-enhancing exchange and polarization-attenuating exchange has been examined by several authors. Very weak encounters $\left(\mathrm{Jt}_{c}<<1\right)$ are unlikely to affect polarization at all $[1,15,17]$. As Syage points out $[17,41]$, most treatments assume this implicitly. His explicit analysis reaches the same conclusion, also reaching the standard finding that encounters of moderate strength $\left(\mathrm{Jt}_{c} \sim 1\right)$ are most effective in promoting RPM. 
Pedersen and Freed [33] found the polarization lost to spin exchange has a dependence on the exchange decay parameter for exchange, $\kappa$, which they expressed as

$$
\Delta P o l \sim\left(r^{\prime} / r_{0}\right)^{1 / 2}
$$

where $\mathrm{r}^{\prime}$ is the radius at which $\mathrm{J}=10^{-5} \mathrm{~J}_{0}$ and $\mathrm{r}_{0}$ is the distance of closest approach for the radical pair. Under conditions of high radical concentration, spin exchange becomes the dominant relaxation mechanism. Adrian has also recently treated spin exchange, making reasonable assumptions about parameters of the stochastic Liouville equations in order to reach analytical solutions [39]. He found that the loss of polarization due to spin exchange for a pair of radicals at distance $r_{a b}$ was given by

$$
\Delta P o l=r_{a b}[(2 \underline{\kappa}+1) / 2 \underline{\kappa}]^{3}=\left(\underline{\kappa} r_{a b}\right)^{-1}\left(.8+\ln \left[64 \underline{J}_{o} / 25 \pi^{2} \underline{\kappa}^{2}\right]\right)
$$

where the underlined parameters have been converted to unitless quantities.

B. $\mathrm{R}_{e x}$ verus $\mathrm{R}_{r \times n}$

Syage predicted that one could relate a characteristic inter-radical distance at which exchange interactions become important $\left(\mathrm{r}_{e x}\right.$, radius of exchange) and the distance at which reactions occurred $\left(r_{r x n}\right.$, essentially $2 r_{o}$ ) to measurable quantities $[17,41]$ :

$$
\left(r_{e x}-r_{r x n}\right) / r_{e x}=1-4 k_{r x n} / k_{e x}
$$

Adrian, as well, noted that the spin exchange cross section can be several times the diffusion-controlled reactive cross section [39].

Recently, the Argonne National Labs group, in collaboration with Lawler and Syage, have made some most exciting measurements in which they obtained approximate values for the radius of spin exchange/radius of reaction ratio for some radicals which recombine at diffusion controlled or nearly diffusion controlled rates $[24,43]$. Pulse radiolysis of $\mathrm{N}_{2} \mathrm{O}$ saturated aqueous solutions of radical precursors produce radicals by the following fast reactions of the primary radicals:

$$
\begin{gathered}
H \cdot+\mathrm{OH}^{-} \rightarrow e_{a q}^{-} \\
\mathrm{N}_{2} \mathrm{O}+e_{a q}^{-} \rightarrow \mathrm{N}_{2}+\mathrm{O}^{-} \\
\mathrm{O}^{-}+\mathrm{RH} \rightarrow \mathrm{OH}^{-}+R
\end{gathered}
$$


where $\mathrm{R}$. is the radical of interest $\left(\cdot \mathrm{CH}_{2} \mathrm{CO}_{2}^{-}, \cdot \mathrm{CH}\left(\mathrm{CO}_{2}^{-}\right)_{2}, \mathrm{CH}_{3} \cdot \mathrm{CHOH}\right.$, $\left.\mathrm{CH}_{3} \cdot \mathrm{CHO}^{-},\left(\mathrm{CH}_{3}\right)_{2} \cdot \mathrm{COH},\left(\mathrm{CH}_{3}\right)_{2} \cdot \mathrm{CO}^{-}\right)$. They suggest that if there is no activation barrier to radical removal on singlet collision, $r_{e x} / r_{r x n}$ should be $1-3$, and find values of $2-5.5$. There is a fair systematic uncertainty in the data (a factor of $c a .1 .5$ ), but the results seem to agree nicely with theoretical expectations.

An interesting result of this work is that $r_{e x} / r_{r x n}$ is substantially larger for the radical anions than for their protonated analogues. This is rationalized by suggesting that $r_{e x}$ is relatively insensitive to charge, but $r_{r x n}$ decreases, presumably because of the addition of the coulombic repulsion.

By analyzing an early account of the $r_{e x} / r_{r x n}$ data [43], and some other data where exchange rates and "diffusion controlled" reaction rates had been determined independently [7,44], Syage compared his model in reference 17 to the numerical solutions of Pedersen and Freed. Good qualitative agreement was found and his equations are much easier to use than the numerical methods [17]. Adrian's equations [39] also yielded self consistent values for the exchange steepness parameter $\kappa$ from data derived for the same radical under substantially different conditions.

\section{Biradical esr spectra}

We turn now to the esr spectra of stable biradicals, such as dinitroxides $[11,15]$. We will consider only biradicals in solvents of low to moderate viscosity so that we may ignore anisotropic interactions, such as the dipole-dipole interactions that dominate typical triplet esr spectra.

The nomenclature of biradical esr can get tricky because there are two phenomena that need to be considered, each of which is an "exchange phenomenon". The first is the effect of singlet-triplet splitting of the unpaired spins, $\mathrm{J}_{\text {mag }}$ res, which dominates the spectra of biradicals in a single, fixed conformation. The second phenomenon is the broadening due to intramolecular spin exchange. Intramolecular spin exchange is important when $\mathrm{J}_{\text {mag }}$ res is strongly modulated by the conformations of the biradical, some with high values of $J$ and others with $J$ less than or approximately zero.

Let us consider the first case: biradicals with a single, relatively fixed conformation. For convenience, the biradicals will be dinitroxides with hyperfine coupling constants $\mathrm{A}$, since most of the solution phase biradical esr literature concerns such molecules. Many nitroxides have simple 3lined esr spectra due to hyperfine coupling with the spin-1 ${ }^{14} \mathrm{~N}$ nucleus, and all other hyperfine interactions will be ignored here. 
Three triplet and one singlet electron spin wave function can be written for the biradical. The two with parallel spins are, as always, $\mathrm{T}_{+}$ and $\mathrm{T}_{-}$, with electron spin configurations of $|\alpha \alpha\rangle$ and $|\beta \beta\rangle$, respectively. The $S$ and $T_{o}$ states are mixed by hyperfine interactions, giving the following wave functions [45]

$$
\begin{aligned}
\Psi^{\prime \prime} S^{\prime \prime} & =\left[2\left(1+\lambda^{2}\right)\right]^{-1 / 2}[(|\alpha \beta>-| \beta \alpha>)+\lambda(|\alpha \beta>+| \beta \alpha>)] \\
& =\left[2\left(1+\lambda^{2}\right)\right]^{-1 / 2}\left[\Psi_{S}+\lambda \Psi_{T o}\right] \\
\Psi_{\prime \prime} T_{o \prime \prime} & =\left[2\left(1-\lambda^{2}\right)\right]^{-1 / 2}[(|\alpha \beta>+| \beta \alpha>)+\lambda(|\alpha \beta>-| \beta \alpha>)] \\
& =\left[2\left(1-\lambda^{2}\right)\right]^{-1 / 2}\left[\Psi_{T o}+\lambda \Psi_{S}\right]
\end{aligned}
$$

where

$$
\lambda=A\left(m_{2}-m_{1}\right) / 2 J
$$

and $m_{i}$ is the nuclear spin state of nucleus $i$. Notice that the mixing disappears as $\mathbf{A}$ goes to zero or as the singlet triplet gap gets too large. Also, for values of $m_{1}=m_{2}$, which accounts for $1 / 3$ of the total population, no mixing occurs. When there is no mixing between the $\mathrm{S}$ and $\mathrm{T}_{o}$ levels, only one transition frequency is observed for each hyperfine line. Thus, in all dinitroxide spectra, the original monoradical lines appear as one-third the total intensity.

When $\lambda \neq 0$, there are four transitions for each hyperfine line corresponding to transitions from each of $T_{+}$and $T_{-}$to each of $\Psi^{\prime \prime} T 0^{\prime \prime}$ and $\Psi$ "S" (known as "triplet transitions" and "singlet transitions", respectively). The frequencies of the transitions are given by

$$
\begin{aligned}
& \omega_{T}=\omega_{0}-(a / 2)\left(m_{1}+m_{2}\right) \pm(1 / 2)\left[J-\left(J^{2}+A^{2}\left[m_{1}-m_{2}\right]^{2}\right)^{1 / 2}\right] \\
& \omega_{S}=\omega_{0}-(a / 2)\left(m_{1}+m_{2}\right) \pm(1 / 2)\left[J+\left(J^{2}+A^{2}\left[m_{1}-m^{2}\right]^{2}\right)^{1 / 2}\right]
\end{aligned}
$$

When $|\mathrm{J}|<<\mathrm{kT}$, the transition probabilities (and hence the relative intensities of the lines) are given by [11]

$$
\begin{aligned}
& P_{T}=(1 / 4)\left[1+J /\left(J^{2}+A^{2}\left[m_{1}-m_{2}\right]^{2}\right)^{1 / 2}\right] \\
& P_{S}=(1 / 4)\left[1-J /\left(J^{2}+A^{2}\left[m_{1}-m_{2}\right]^{2}\right)^{1 / 2}\right]
\end{aligned}
$$

where the total intensity has been normalized to 1 , and the subscripts $S$ and $T$ refer to singlet and triplet transitions in both Eqs. (32) and (33).

Let us examine the behavior of the spectrum at the limits of high and low J (Figure 7). As J goes to $0, \Psi_{\prime \prime T o^{\prime \prime}}$ and $\Psi_{\text {" }} S^{\prime \prime}$ become degenerate, $\mathrm{P}_{T}=\mathrm{P}_{S}$, and the biradical spectrum looks identical to that of the two 


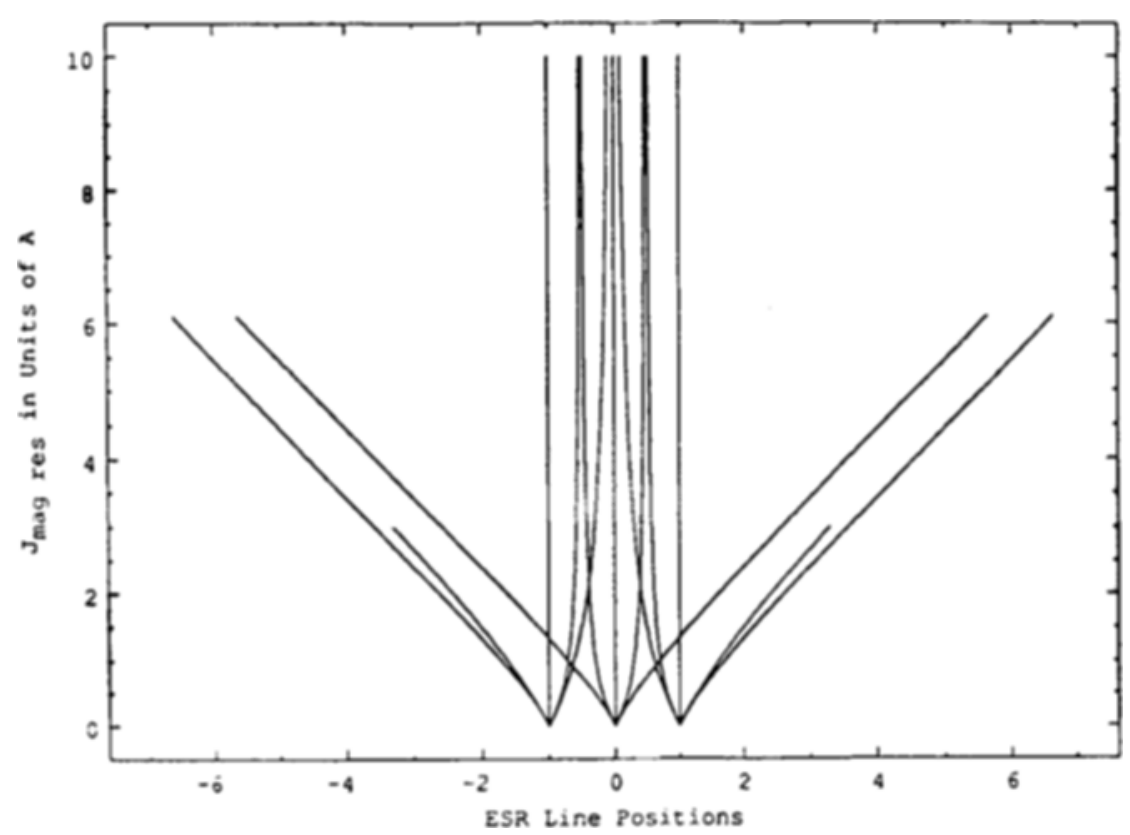

Figure 7. The esr spectrum of nitroxide biradicals as a function of J. The horizontal axis is in units of the hyperfine interaction $\mathrm{A}$. With $\mathrm{J}=0$, the typical 3-lined spectrum is observed. With $\mathrm{J}>\mathrm{A}$, a 5 -lined spectrum with intensity ratios of $1: 2: 3: 2: 1$ is observed. In intermediate ranges, the spectrum is complex, but $|\mathrm{J} / \mathrm{A}|$ can be directly determined. In the figure, lines are no longer shown when their intensity is $<5 \%$ of the intensity at $\mathrm{J}=0$.

monoradicals, as would be expected for a pair of non-interacting identical radicals. If $|\mathrm{J}| \gg|\mathrm{A}|$, then $\mathrm{P}_{S}$ goes to $0, \lambda$ goes to 0 , and the spectrum collapses to a five line spectrum with apparent hyperfine splitting of $A / 2$ and an intensity pattern of 1:2:3:2:1 due to the interaction with two spin $=1\left({ }^{14} \mathrm{~N}\right)$ nuclei. When $|\mathrm{J}|$ is very large, the electrons are interacting very strongly; it is no longer valid to consider the biradical as a pair of doublets. Instead the electron spin functions must be taken over both nitroxide centers and one can think of the electron of spending half of its time on each nucleus.

If, however, $|\mathrm{J}| \sim \mathrm{A}$, then the spectrum is clearly quite complex. Fortunately, though, the equations presented in this section allow for a direct measurement of $|\mathrm{J}| / \mathrm{A}$, thus giving $|\mathrm{J}|$ ! 
Consider another biradical, one with two or more slowly interconverting conformations. In such a case, the observed spectrum is a superposition of two or more spectra of the sort just discussed. Now let us assume that the biradical can assume more than one conformation and the interconversion can be fast. In each conformation (or small range of conformations), we assume that $\mathrm{J}$ has some discreet value. As in the single conformation case, the monoradical spectrum appears with one-third the total intensity, regardless of exchange interactions. If the conformational interchange is sufficiently rapid, then a spectrum based on a weighted average $\langle\mathrm{J}\rangle$ is observed. This spectrum follows the equations already set forth in this section. However, the rapid equilibration of conformations is readily distinguishable from a single conformation spectrum via its temperature dependence. A spectrum representing an averaged $<\mathrm{J}>$ will change with temperature, since the conformational equilibria will change. As long as $\langle\mathrm{J}\rangle \sim \mathrm{A}$, this will show up as changes in line position and intensity.

In cases where $\langle\mathrm{J}\rangle>>\mathrm{A}$, frequency and intensity changes are not visible with moderate changes with temperature (see Figure 7). However, intramolecular spin exchange broadens the biradical components of the spectrum (i.e., those components where $\mathrm{m}_{1} \neq \mathrm{m}_{2}$ ), while the monoradical portions remains sharp. As long as the time between the collisions, $t_{o}$ (the time the molecule is not in the high $\mathrm{J}$ conformation) is much greater than the collision time, $\mathrm{t}_{c}$, the frequency of exchange is determined by exactly the same equations as in section IIB, giving

$$
\omega_{\varepsilon}=\left(1 / 2 t_{o}\right)\left(J^{2} t_{c}^{2} /\left[1+J^{2} t_{c}^{2}\right]\right)
$$

Under conditions of strong exchange, the $m_{1} \neq m_{2}$ components of the central line have a width of $1 / T_{2}+A^{2} / 2 \omega_{e}$. The half-A lines have widths of $1 / T_{2}+A^{2} / 8 \omega_{e}[11,15]$. However, when conformational interchange $\left(\omega_{c}\right)$ is very fast (i.e., $\left.\omega_{c}>>|J|>>A\right), t_{c}$ is limited to be $\omega_{c}^{-1}$, and no broadening is observed. This situation is analogous to the rapid exchange limit of monoradicals (where exchange narrowing occurs) and could be called the case of rapid (conformational), strong (electronic) spin exchange.

In practice, of course, the simplifying assumptions made here do not always apply. Very often, multiple conformations with $|\mathrm{J}| \sim \mathrm{A}$ are available, leading to very complex spectra. The rate of conformational exchange affects these spectra as well. The reader should see reference 11 for a detailed treatment. Suffice it to say here that temperature dependences of the spectra can lead to determinations of the minimum number of conformations needed to explain the spectra, the effective $J$ for each 
conformation, and some thermodynamic data about the conformations' stabilities.

Having gone through this whole development, it is instructive to look at some of the values of $\mathrm{J}$ that have been obtained. Table II shows the

\section{TABLE II}

$\mathrm{J}_{\text {mag res }}$ for dinitroxide biradicals. $A=15.3, \mathrm{G}=48.3 \mathrm{MHz}$. Data taken from reference 11 .

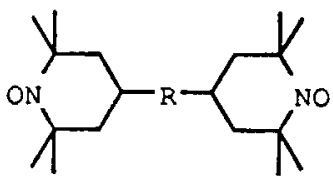

$$
\mathrm{R}=\quad|\mathrm{J} / \mathrm{A}|
$$<smiles>NC(=O)O</smiles>

$0^{-5} \mathrm{O}$

0.4<smiles>O=[SH](=O)O</smiles><smiles>[2H]S(=O)(=O)O</smiles>

웅

웅

$0-\mathbb{P}_{1}^{-0}$

$0-0$
$0-0$ 
values of $|\mathrm{J}|$ ranging from 3.4 to $25 \mathrm{MHz}$ obtained for TEMPO-type biradicals with short connecting groups. These bridge groups do not allow the radical fragments to approach to collision distance. Moreover, the constants are essentially independent of temperature and solvent. Temperature independence suggests that conformational interchange plays little role in the value of $J$ and independence from solvent suggests that exchange is through bond, rather than through space. Notice also that the $J$ values are fairly constant with the type of interconnecting atom with varying substitution. The idea of through bond coupling through so many $\sigma$-bonds is also found in the electron transfer literature (see section IIIF).

Recently, Eaton and Eaton reviewed their work on long-range electron-electron exchange interactions [46]. Most of the compounds they have prepared and studied over the last decade are transition metal complexes $(\mathrm{M}=\mathrm{VO}, \mathrm{Cu}, \mathrm{Ag}, \mathrm{Cr})$ with spin labeled pyridines, such as in Figure 8, as one of the ligands. By varying the metal, the bridging groups between the spin label and the pyridine moiety, and the geometry of the spin label relative to the metal, values of $|\mathrm{J}|$ were obtained that ranged from $100 \mathrm{MHz}$ to $5.6 \mathrm{GHz}$ ! Such large values of $\mathrm{J}$ are not so surprising when one reconsiders Eq. (4) and realizes how different $\chi_{1}$ and $\chi_{2}$ are.
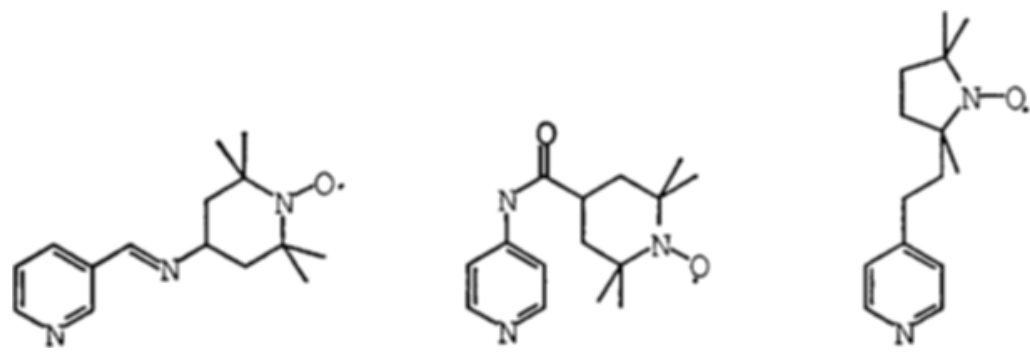

Figure 8. Some of the spin-labeled ligands used by Eaton, Eaton, and coworkers.

They found that spin delocalization decreased over the metal series $\mathrm{Ag}(\mathrm{II})>\mathrm{Cu}(\mathrm{II})>\mathrm{VO}(\mathrm{II})$ and that the geometry of the bridging groups was important, with "W-shaped" giving the largest couplings. Other interesting effects included NO complexing to metal orbitals in unsaturated complexes leading to huge coupling which could be greatly diminished by displacing the nitroxide with complexing solvents (e.g. pyridine). Frozen solution studies in the same system showed that the decrease in $\mathrm{J}$ was accompanied by an increase of about $2 \AA$ in $\mathrm{M}-\mathrm{NO}$ distance. 


\section{Biradical and correlated radical pair CIDEP}

In 1984, Sakaguchi, et al. published time-resolved esr spectra obtained from laser photolysis of micellar solutions of napthoquinone and benzophenone [47]. At early times, (ca. $1 \mu \mathrm{s}$ post-photolysis) each of the lines which could be attributed to the respective ketyl and surfactant radicals appeared in an E/A pattern. At somewhat longer delays (ca. $3 \mu$ s post-photolysis and later), the spectrum returned to a more standard-looking all emissive spectrum attributed to triplet mechanism (TM). Figure 9 shows spectra collected under similar conditions.

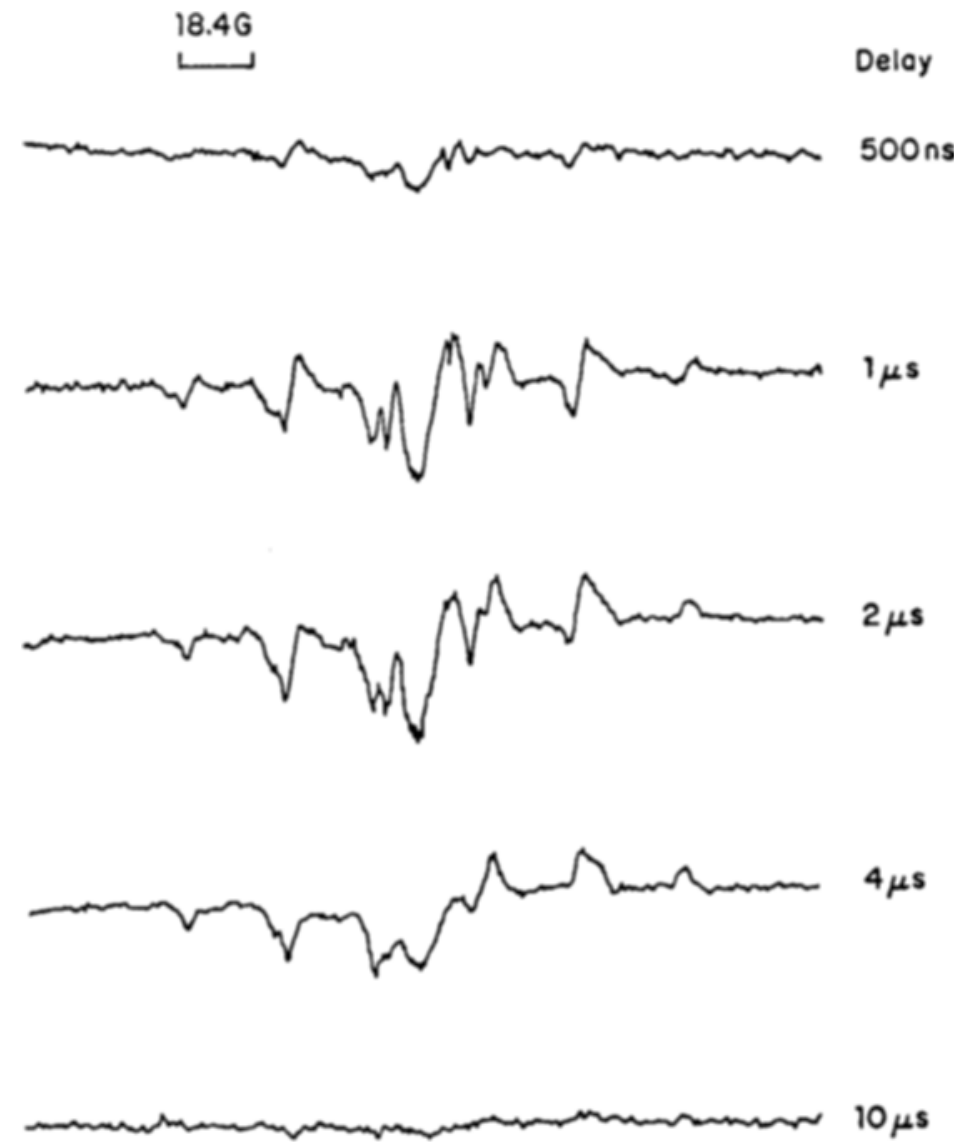

Figure 9. Time resolved esr spectra obtained on photolysis of aqueous micellar solutions containing benzophenone at various delays. 
As Sakaguchi pointed out, the early spectra are incompatible with any of the standard CIDEP polarization mechanisms. Several other publications by the Japanese group and by McLauchlan's group at Oxford confirmed the "antiphase" lines for hydrogen abstraction reactions in micelles at short delays and different explanations for the effect were offered [48]. Similar spectra had actually been reported as early as 1977 without special comment from pulse radiolysis of micellar solutions [49]. However, the signal-to-noise ratio of these earlier spectra were such that one might have been skeptical about the antiphase nature of the apparent hyperfine lines.

But in 1987 the Oxford group [50] and Closs, et al. [51] at the Argonne labs published what has become the accepted explanation for the appearance of the antiphase lines. The polarization is not related to either RPM or TM, but arises directly from taking the esr spectrum of a spin correlated radical pair. The basic mechanism is very similar to the biradical spectra as discussed in section IIIC but depends on the selective depletion of certain levels to give emissive and absorptive lines. Figure 10 (adapted from references 51 and 52) shows a diagram for one of the hyperfine combinations. The four transitions outlined in section IIIC are marked.

The radical pair is assumed to be born of a triplet precursor with the triplet sublevels equally populated. As shown in the figure, the $\Psi_{S}$ and $\Psi_{\text {To }}$ states are initially separated by $\mathrm{J}[53]$. Hyperfine mixing separates the levels further to $2 \omega=\left(\mathrm{J}^{2} / 4+\mathrm{Q}^{2}\right)^{1 / 2}$, and tends to equalize populations of the $T_{o}$ and $S$ levels. It is assumed that the populations of the four levels are depleted due to reaction in the order of $\Psi_{S}>\Psi_{T o}>\Psi_{T-}$ $>\Psi_{T+}$. This gives rise to the observed polarization because transitions involving $T_{+}$and $\Psi_{S}$ or $\Psi_{T o}$ will be emissive, while those involving $\mathrm{T}_{-}$ and $\Psi_{S}$ or $\Psi_{T o}$ will be absorptive. Thus a doublet of doublets is observed. When $\mathrm{J}<\mathrm{Q}$, the large splitting is twice the hyperfine for the pair in most cases (i.e., when $\mathrm{Q}$ is hyperfine-dominated). Each of these two lines is further split into a doublet of width $\mathrm{J}$, with the low field line in emission, and the high field line in absorption. Thus $\mathrm{J}$ can be measured directly from simulations of the spectra!

The observed polarization from this mechanism is largest when $\mathrm{J}<\sim$ Q. If $\mathrm{J}$ is too small, the $\mathrm{E}$ and $\mathrm{A}$ lines overlap each other, cancelling out the polarization and therefore giving essentially no spectrum. This, of course, corresponds to the case where the radical pair is no longer correlated. When $\mathrm{J}>>$ Q the reaction rate from $\Psi_{T o}$ is not high because the $\Psi_{S}$ and $\Psi_{T o}$ levels are not well mixed. Thus, transitions involving $\Psi_{T o}$ do not 


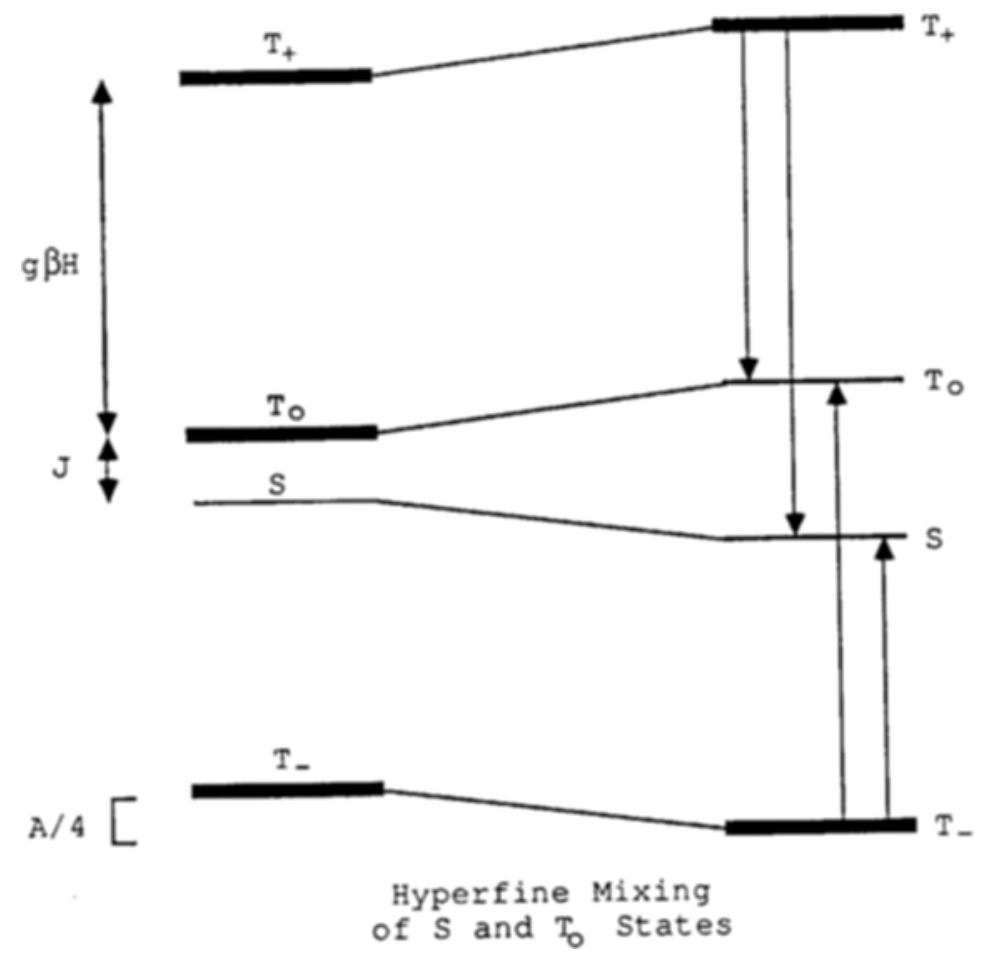

Figure 10. A schematic diagram of the "biradical" mechanism of CIDEP. This diagram is for one particular hyperfine combination. The thickness of the energy levels represents their relative populations. The biradicals are born with equal distribution in all three triplet sublevels. After hyperfine mixing, the levels split and the populations of $S$ and $T_{o}$ are equalized. $S$ and $T_{o}$ are depopulated by reaction, so transitions are absorptive or emissive as indicated by the arrows.

show large polarization. Moreover, transitions involving $\Psi_{S}$ are largely forbidden.

The polarization pattern of "early" spectra observed for the micellar solutions $[47,48]$ is now understood to be due to this biradical mechanism. Later spectra obey the previous RPM and TM (if the triplet sublevels are not equally populated) rules when the radical pairs are no longer correlated. Two pathways lead to the loss of correlation. The first is singlet reaction (recombination, disproportionation, etc.) and the second is escape of radicals from the micelle. When the ketyl escapes, the interradical 
distance is large enough that the radicals become uncorrelated. The "normal polarization" spectra are observed a few microseconds after radical creation, which is very consistent with typical rates of radical exit from the micelle measured by other means [54]. To the best of these authors' knowledge, no one has undertaken a systematic study of the change from biradical mechanism to TM or RPM versus known or otherwise measured radical exit rates in micellar solution.

Closs and Forbes extended the biradical mechanism idea by measuring TRESR spectra for photochemically generated polymethylene biradicals of the sort shown in Figure 11. Their simulations of the spectra were very satisfactory, yielding values of $|\mathrm{J}|$ ranging from $2.1 \times 10^{8} \mathrm{~Hz}$ for $\mathrm{n}$ $=10$ to $1.1 \times 10^{9} \mathrm{~Hz}$ for $\mathbf{n}=8$. They were also able to observe the nondecarbonylated (i.e., alkyl-acyl) radicals and measure $\mathrm{J}$ for in these cases. These biradicals are flexible, and thus $\mathrm{J}$ depends on the conformation. The value observed here and with the micellar radical pairs represents a time-weighted average over the values of $J$ in all conformations.
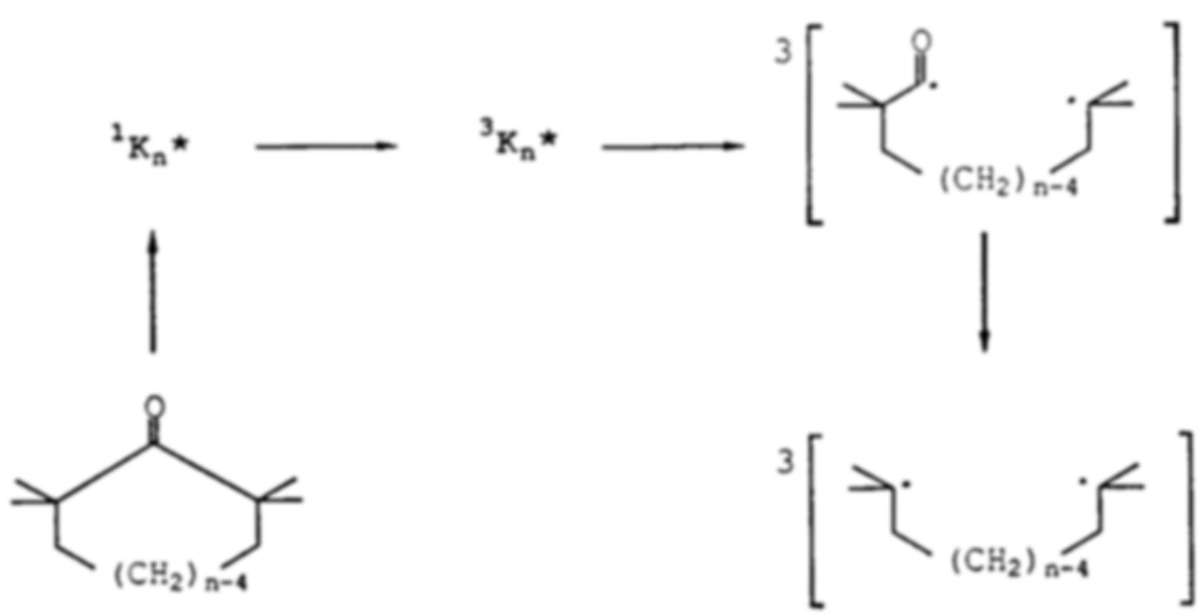

$\mathrm{K}_{\mathrm{n}}$

Figure 11. The biradical system used by Closs and Forbes.

The biradicals of Closs and Forbes should fall within the rapidly equilibrating multi-conformational biradical case discussed in section IIIC. If so, as long as the temperature is high enough to give rapid decarbonylation, the measured J should be a function of temperature. The dinitroxide results would tend to suggest that $\mathrm{J}$ should increase with temperature, 
since the more stable conformation is an extended one with larger interradical separation and lower $J$. Their experiments were carried out from $-10^{\circ}$ to $60^{\circ} \mathrm{C}$, but $\mathrm{J}$ was not reported as a function of temperature for any one biradical, but unpublished experiments confirm this prediction [55]. Similar phenomena have recently been observed by Wang, et al. who observed longer biradical lifetimes at higher temperatures [56]. The longer lifetimes are due to intersystem crossing which is less efficient at high J because hyperfine mixing is the dominant mechanism.

A similar phenomenon resulting in out of phase signals in electron spin echo (ESE) spectra was actually observed several years before the work of Sakaguchi, et al. when Thurnauer and Norris obtained very fast time-resolution of a photosynthetic algae system [57]. Using a laser- $\mathrm{t}_{1-}$ $90^{\circ}-t_{2}-180^{\circ}$ pulse sequence, they observed a phase shift for a $g=2.0023$ signal on the $t_{1}=30-40 \mathrm{~ns}$ timescale. They showed how this could arise from a "biradical mechanism" if the radical pair correlation disappears during $t_{2}$.

Another method for measuring $\mathrm{J}$ of radical pairs (that has mostly been applied to photosynthetic systems) is RYDMR, or Reaction Yield Detected Magnetic Resonance [58], first used by Frankevich and Pristupa [59]. In its form most similar to "normal" time resolved esr, a very high microwave field is applied to the cavity, and the laser is repetitively fired as usual while the magnetic field is swept. However, instead of detecting microwave absorption, product yield (often in the form of fluorescence or phosphorescence) is detected $[1 c, 58]$. This allows for detection at delays much shorter than available with the standard experiment because it avoids the relatively long dead times (usually $>100 \mathrm{~ns}$ ) associated with the normal est detection. If we assume that a radical pair is born in the singlet state, and phosphorescence is monitored, then when esr transitions come into resonance as the field sweeps, the phosphorescence will increase, since intersystem crossing is increased by stimulation of the $T_{+} \leftrightarrow S$ and $\mathrm{T}_{-} \leftrightarrow \mathrm{S}$ transitions. In the very fast experiment, uncertainty broadening wipes out any fine structure regardless of detection method, but the intensity of the RYDMR as a function of microwave power is related simply to the coupling constant J. At longer delays, the structure returns if the coherence of the radical pair remains.

Another relatively convenient way to measure the singlet triplet gap in correlated radical pairs is by low field CIDNP [60]. The principle here is simple. Again consider a correlated radical pair or biradical, but at zero magnetic field. If we assume that the singlet lies below the triplet, then as the magnetic field is raised, there will come a point where the $S$ and $\mathrm{T}_{-}$levels will be degenerate. This is also where the most efficient $\mathrm{S} / \mathrm{T}_{-}$ 
mixing will occur and is the same energy as $\mathrm{J}_{\text {mag res }}$ in the absence of a magnetic field (neglecting $Q$ ). Thus, if the radical precursor is photolyzed in a variable (low) magnetic field and then quickly transferred to an NMR where the S/T _ CIDNP is measured, the CIDNP should show a maximum at the magnetic field yielding $\mathrm{g} \beta \mathrm{H}=\Delta \mathrm{E}_{T S}$. Similarly, $\mathrm{S} / \mathrm{T}_{-}$crossings can be observed by following triplet decay rates as a function of applied field, where the magnetic field with the maximum decay rate is traditionally interpreted as the "J resonance" or $\mathrm{S} / \mathrm{T}_{-}$crossing $[58,61]$. These results refer to the biradicals in their extended conformations because intersystem crossing is dominated by spin-orbit coupling at small interradical distances and intersystem crossing is occuring in the extended conformations due to hyperfine interactions.

Low field NMR also yields the sign of $\mathrm{E}_{S T}$ since ST_ CIDNP yield emissive signals, whereas $\mathrm{S} / \mathrm{T}_{+}$mixing yields enhanced absorption. Zimmt, et al. used this technique to measure the average $\mathrm{J}$ of benzylbenzyl radical pairs generated in micelles by photolysis of dibenzyl ketone and found values of the order of $10^{9} \mathrm{~Hz}$ [62]. The value of $\mathrm{J}$ was inversely related to the length of the surfactant, consistent with the idea that the radicals could further separate in a larger micelle.

\section{E. Spin exchange-induced intersystem crossing}

The literature is filled with references to intersystem crossing "catalyzed" by paramagnetic species. For instance, oxygen and nitroxides are both efficient singlet and triplet quenchers. Often these quenching "reactions" involve energy transfer, which will be discussed in section IIIF. Many groups have studied increases and/or decreases in fluorescence and/or phosphorescence as a function of added paramagnetic material and have attributed changes to "catalyzed" intersystem crossing. Others have used product yields as probes when singlet and triplet products can be distinguished. Usually, though, there is very little "hard" data on the mechanism of the increased intersystem crossing. Recently, work at Columbia has shown that intersystem crossing of triplet biradicals can be accelerated by paramagnetic lanthanide ions, specifically by spin exchange [63].

Some hint that spin exchange mediated intersystem crossing could be important for triplet biradicals came from the work of Scaiano and Encinas [64] and Creed and Caldwell [65]. Scaiano and Encinas measured product yields of the photolysis of various valerophenone derivatives (Figure 12) as a function of added di-t-butylnitroxide (DTBN). Based on kinetic arguments, they concluded that the DTBN was interacting with the 


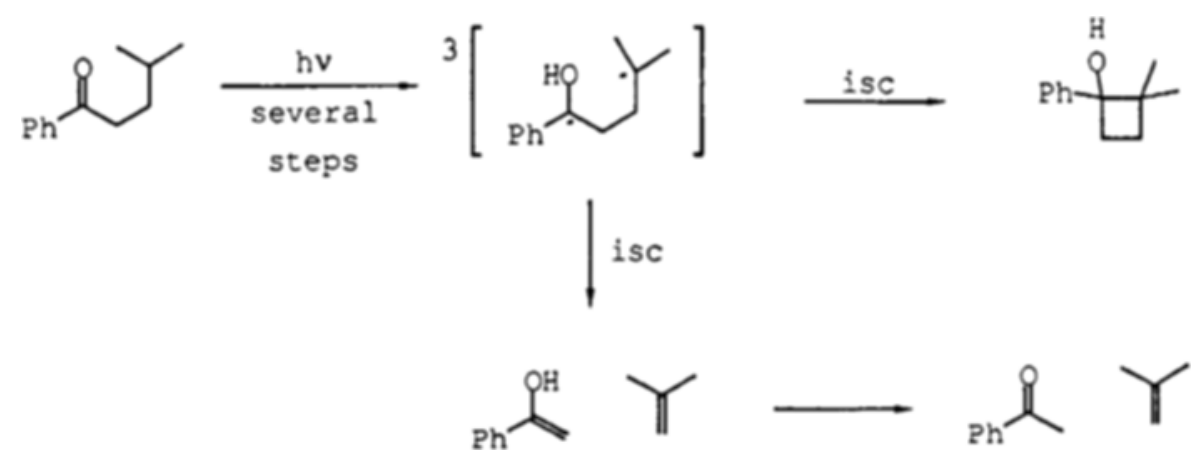

Figure 12. A scheme of the photolysis of $\gamma$-methylvalerophenone. The triplet biradical must undergo intersystem crossing before forming products. Scaiano, et al. found that addition of lanthanides changed the product distribution via interaction with the biradical. See text for discussion.

triplet 1,4-biradical, rather than the excited triplet ketone. Intersystem crossing is generally thought to be the rate limiting step in the disappearance of these triplet biradicals, and it was observed that increasing the DTBN concentration increased the quantum yield of acetophenone and isobutylene without affecting $\Phi_{T}$. This, along with Arrhenius rate data, was taken to suggest that there was a conformation-specificity for the induced intersystem crossing. A similar explanation had been invoked earlier by Creed and Caldwell using only product study data [65]. Since the apparently favored conformation was the one with the radical centers most separated from each other, it is reasonable to speculate the accelerated intersystem crossing occurred when $\mathrm{J}_{\text {mag }}$ res was minimized. These results are consistent with both dipole-dipole and spin-exchange interactions between DTBN and the biradical.

The addition of nitroxides to solutions of large fused aromatic hydrocarbons, such as pyrene, has been shown to increase the quantum yield of excited aromatic triplet formation on excitation $[66,67]$. The proposed explanation for this effect is that the nitroxide interacts with the aromatic in its excited singlet state, assisting the otherwise relatively forbidden intersystem crossing process. No evidence exists as of yet as to the specific mechanism for this effect, although spin exchange is certainly reasonable. These systems are discussed further in section IIIF.

Specific evidence for spin-exchange induced intersystem crossing comes from the dynamics of interaction between a 1,9-polymethylene biradical and lanthanide ions [63]. In the absence of magnetic fields, lan- 
thanides, or any other additives, the rate limiting step in the decay of biradical is held to be intersystem crossing from the triplet to the singlet (Figure 13). Wang, et al. found that the addition of certain lanthanide ions $\left(\mathrm{LnCl}_{3}\right)$ to methanolic solutions decreased the lifetime of the biradical. Figure 14 shows the second order quenching rate constants that were measured for the various lanthanides.
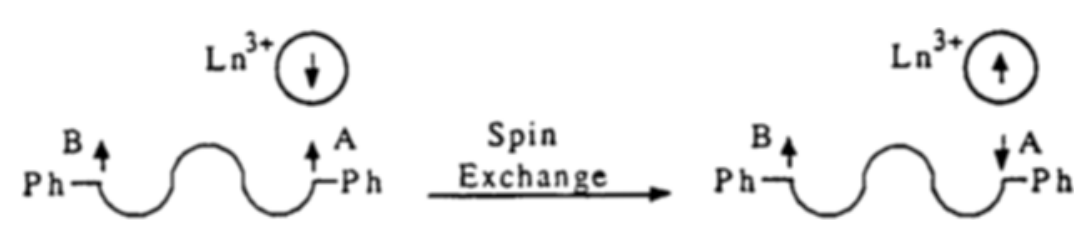

Figure 13. Spin exchange induced intersystem crossing of a triplet biradical by a lanthanide ion.

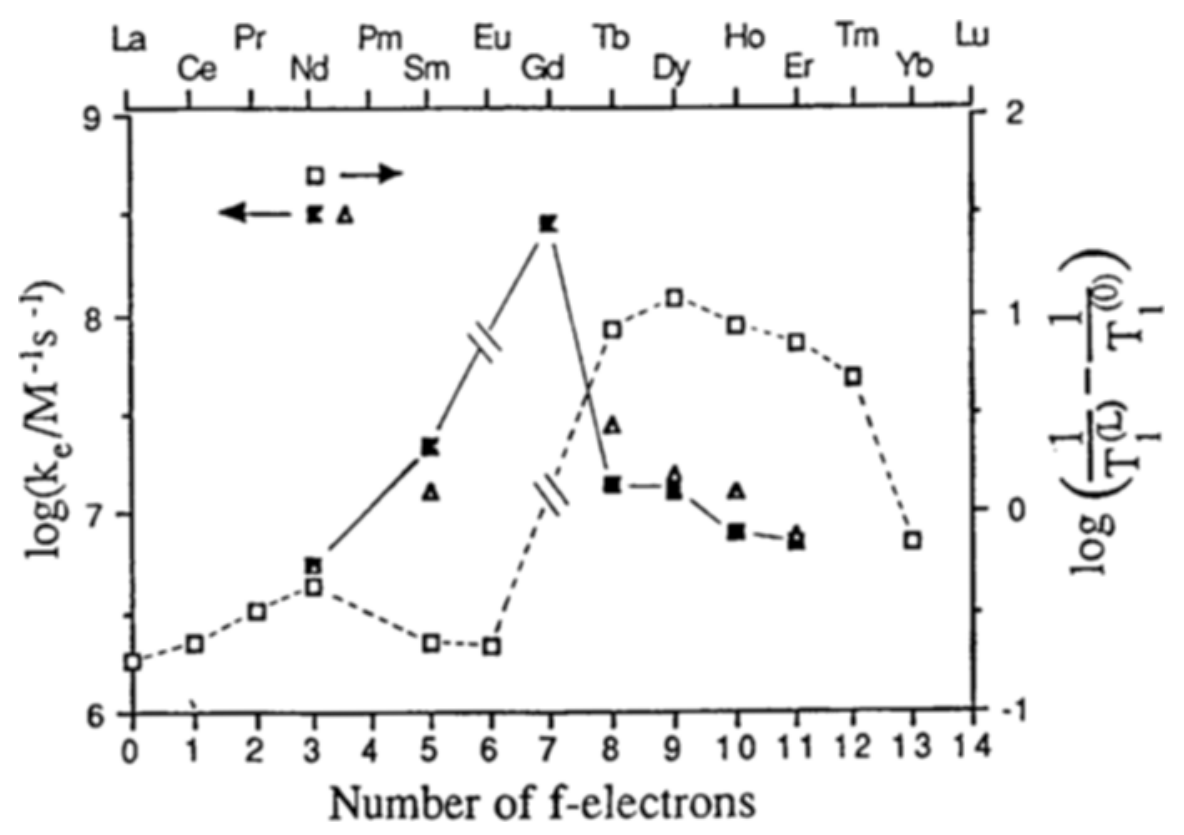

Figure 14. ( - ): non-zero $k_{e x}$ values; $(\Delta)$ ): relative calculated $k_{e x}$ values normalized by $\mathrm{Nd}^{3+} ;(\mathrm{a})$ : proton relaxation enhancements due to dipole interactions. The left ordinate refers to the $\mathrm{k}_{e x}$ values and the right refers to the nuclear relaxation. 
The quenching mechanism was assigned to be spin exchange for the following reasons. (1) The quenching is not due to chemical reactions (no new products). (2) Rapid reversible electron transfer is not compatible with the redox potentials of the lanthanides [68] and 1-phenylethyl [69] (which is taken as a model for the biradical). (3) Dipolar relaxation is inconsistent with the pattern of $\mathrm{k}_{e}$ versus $\mathrm{Ln}^{3+}$. (4) The rate pattern is in qualitative agreement with the expectation for spin exchange.

Lanthanide shift reagents are commonly used in NMR and the dipolar interaction is typically the most important [63]. An NMR $T_{1}$ experiment in which the enhanced relaxation of the $\alpha$-protons of sodium dodecylsulfate (SDS) molecules was measured as a function of $\mathrm{Ln}^{3+}$ showed a totally different pattern than the biradical quenching rates (Figure 14). The measured quenching rates of the biradicals by $\mathrm{LnCl}_{3}$ were well below diffusion the diffusion controlled limit, suggesting that spin exchange would be in weak exchange limit $\left(\mathrm{J}^{2} \mathrm{t}_{c}{ }^{2}<<1\right)$. In the weak exchange limit, the spin exchange rate between a lanthanide and a biradical should be given by [63]

$$
\begin{array}{cc}
k_{e}=\left[f\left(g_{J}-1\right)^{2} L(L+1) J^{2} t_{c}{ }^{2}\right] k_{d i f f} & t_{c}<T_{1 e} \\
k_{e}=\left[f\left(g_{J}-1\right)^{2} L(L+1) J^{2} t_{c} T_{1 e}\right] k_{d i f f} & t_{c}>T_{1 e}
\end{array}
$$

where $f$ is the steric factor discussed in section IIB, $g_{J}$ is the Lande $g$ factor, and $L$ is is the total angular momentum of the lanthanide ion [70]. Assuming that $f, J$, and $t_{c} T_{1 e}$ are relatively constant within the series of lanthanides, except for $\mathrm{Gd}^{3+}, \mathrm{k}_{e x}$ should be proportional to $\left(\mathrm{g}_{J}-1\right)^{2} \mathrm{~L}(\mathrm{~L}$ $+1)$. Those points, scaled to the rate for $\mathrm{Nd}^{3+}$ are also given in Figure 14. $\mathrm{Gd}^{3+}$ has a substantially longer relaxation time, so equation (35a) is applicable because $t_{c}<T_{1 e}$. The value of $T_{1 e}$ is about $3 \times 10^{-9} \mathrm{~s}$; this is in a reasonable range to give the factor of 13-50 rate enhancement.

Three groups have found that magnetic field effects for micellar radical pairs can be wiped out by the presence of lanthanide ions, but the evidence tying the effect to spin exchange is tenuous. Sakaguchi and Hayashi studied the behavior of radical pairs created by hydrogen abstraction from a micelle by triplet naphthoquinone [71]. Monitoring the optical absorption of the naphthosemiquinone radical, they found a two component decay, which was attributed to fast recombination within the micelle and slow reaction outside the micelle. Once again, it was assumed that intersystem crossing of the initially triplet radical pair to the singlet was the rate determining step for the fast decay.

On the application of a magnetic field of $10 \mathrm{kG}$, they found that the ratio of (escaped semiquinone radicals/recombined semiquinone radicals) 
rose from about 0.3 to about 0.7 , based on optical density. The standard explanation for such a magnetic field effect is that intersystem crossing between $\mathrm{T}_{+}$or $\mathrm{T}_{-}$and $\mathrm{S}$ is cut off when the degeneracy is lifted by the Zeeman interaction. The magnetic field effect could be washed out by the addition of paramagnetic lanthanide salts to the micellar solutions. Remarkably, the lanthanides did not substantially alter the radical pair dynamics at zero magnetic field; the effect required the application of a modest magnetic field $(<1000 \mathrm{G})$ to be observed. Such results suggested that in the presence of the magnetic field, the lanthanides induced relaxation between $T_{+}$or $T_{-}$and $T_{o}$, from whence intersystem crossing is reasonably efficient. (In the absence of the magnetic field, $\mathrm{T}_{+}$or $\mathrm{T}_{-}$ and $T_{o}$ are no longer even proper descriptions of the triplet sublevels.) The rate of the lanthanide induced relaxation was not dependent on the magnetic susceptibility (as would be expected for dipole interactions), but rather the spin only moments.

A similar correlation was found by Turro, et al. for the lanthanide effect on the magnetic field effect on product distributions in micelles [72]. On photolysis of $p$-methyl dibenzylketone $(\mathrm{A}-\mathrm{CO}-\mathrm{B})$ in a micelle, a non-zero cage effect, defined as

$$
C . E .=(A B-[A A+B B]) /(A A+A B+B B)
$$

is obtained. In the absence of a magnetic field, the addition of lanthanides had no effect on the product distribution that could not be attributed to salt effects. On the other hand, the cage effect increased when lanthanides were added to micellar solutions in a magnetic field. A correlation between the magnitude of the lanthanide effect and the spin-only moment was found in this case as well, and the results were interpreted in a very similar fashion as the Japanese researchers.

Basu, et al., found a third example using exciplex quenching in the presence of a magnetic field [73]. Their lanthanides were in the form of $\operatorname{Ln}(\mathrm{acac})_{3}$ complexes and they found a very similar rate pattern to that seen for the two previous systems. They used qualitative arguments about the order of effectiveness to eliminate dipole interactions, other multipole interactions, and virtual phonon exchange, thus giving spin exchange by process of elimination.

By analogy to some magnetic transitions in lanthanum alloys in which spin exchange is important, Basu, et al. suggest that the rates for the various lanthanide complexes ought to be proportional to $\mathrm{G}^{1 / 2}=\left(\mathrm{g}_{J}-1\right)$ $[\mathrm{L}(\mathrm{L}+1)]^{1 / 2}$, or the square root of what Wang, et al. had suggested. Very recently, Basu has considered this conflict but has yet to find a resolution [74]. She found that, of several different fittings, her data best fit her 
original suggestion. However, she points out that between the limits of weak and strong exchange, the exponent of $G$ can vary from 0 to 1 .

All groups agree that the washing out of magnetic field effects by lanthanide ions is some form of induced relaxation mechanism [75]. While dipole interactions have been ruled out in some cases, the details of that relaxation are still an open question.

\section{F. Energy transfer, electron transfer, and CIDEP}

In this section, we will examine two energy transfer mechanisms and look at their relationship to spin exchange in terms of spin polarization transfer. The spin polarization we are speaking of is that which also causes standard triplet mechanism, i.e. sublevel selective intersystem crossing from an excited singlet to a triplet state, leading to non-Boltzmann spin distributions among the triplet sublevels. Thus, we will be interested in energy transfer where the donor is a triplet. We will consider both singlet and doublet acceptors. A review of time-resolved esr of intersystem crossing and energy transfer processes, with particular emphasis on direct observation of triplets at low temperature, has appeared recently in this journal [76] so we will concentrate more on the ideas involved rather than try to give an exhaustive review of the literature.

Three mechanisms typically come to mind when energy transfer in solution is considered [77]. The first of these is the so-called "trivial" mechanism in which the excited molecule emits a photon (phosphoresces, since we are interested in triplets) and, in a totally uncorrelated event, an acceptor molecule absorbs the photon. While this can be an important energy transfer mechanism, there is no interaction between the electrons of the donor and acceptor, so no polarization can be transferred by direct electronic interactions. Thus we will not consider this mechanism further.

The two other mechanisms for electronic energy transfer are the dipole-dipole (Förster [78]) and exchange-mediated (Dexter [79]) mechanisms. Perhaps it is given away simply by their names, but we will see through a simple pictorial model below that only the latter is in any way analogous to spin exchange and that only by exchange mediated energy transfer can electron spin polarizaton be transferred as well.

Figure 15 is a simple pictorial representation of dipole-dipole (DD) and exchange-mediated (EM) energy transfer without any consideration of spin. Of course electrons are indistinguishable, so one could not experimentally show this, but the key difference is that in the EM mechanism an electron from each molecule "switches places" with another electron on the other molecule, whereas with DD mechanism, the through-space 


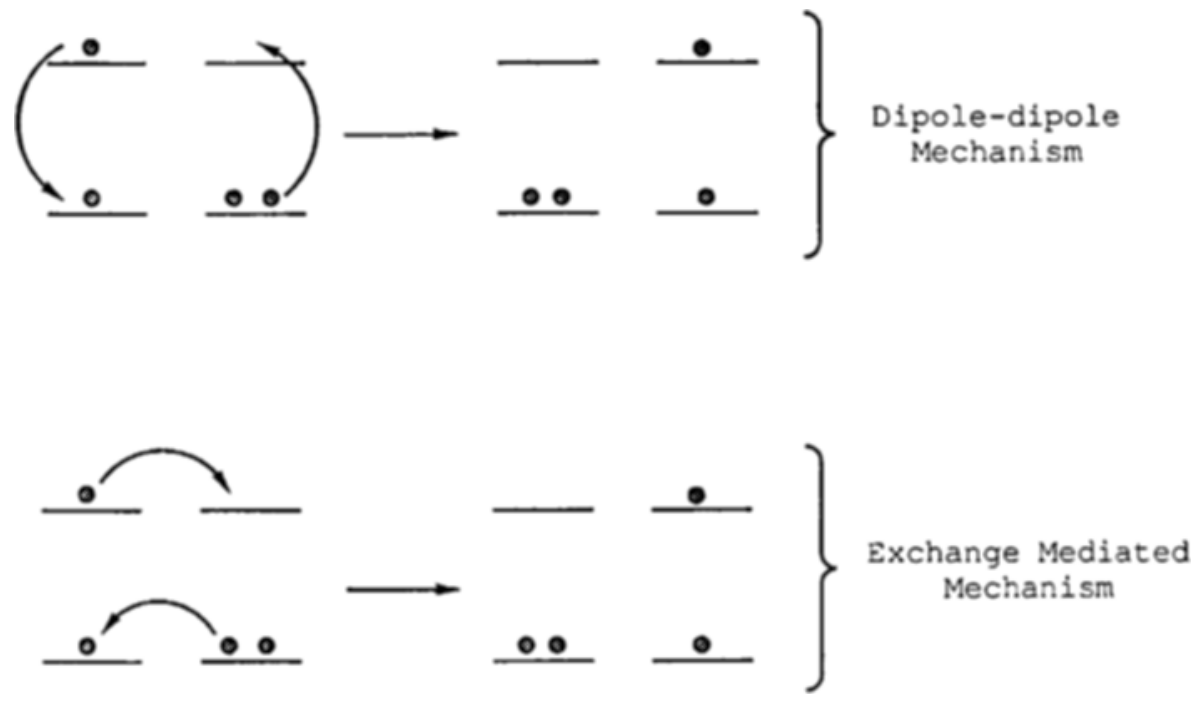

Figure 15. A schematic representation of the dipole-dipole and exchange mediated energy transfer mechanisms without reference to spin.

interaction causes electrons on each molecule to promote or demote themselves on the same molecule.

We are interested in spin polarization, so let us consider the spin requirements for both DD and EM energy transfer. The spin requirement for DD is that [79]

$$
<\chi_{d i}\left(\sigma_{1}\right)\left|\chi_{d f}\left(\sigma_{1}\right)><\chi_{a i}\left(\sigma_{2}\right)\right| \chi_{a f}\left(\sigma_{2}\right)>\neq 0
$$

where $\chi_{d i}\left(\sigma_{1}\right)$ is the spin function of the electron on the donor in the initial state. Stated in words, the requirement is that there should be no spin flips on either the donor or the acceptor.

In contrast, the spin requirement for EM is [79]

$$
<\chi_{d i}\left(\sigma_{1}\right)\left|\chi_{a f}\left(\sigma_{1}\right)><\chi_{a i}\left(\sigma_{2}\right)\right| \chi_{d f}\left(\sigma_{2}\right)>\neq 0
$$

Stated in words, the requirement is that the initial spin of each "moving" electron is the same as its final state on the other molecule. But although total electron spin is preserved, notice that there is no requirement for the spin state of the electron on the donor (or the acceptor) to be the same before and after energy transfer. In other words, the spin functions can be exchanged! 
Another requirement for efficient DD energy transfer is high oscillator strengths for the two transitions. Since $S_{o}$ to $T_{n}$ absorptions are almost always very weak and phosphorescence is associated with weak oscillator strength, it generally held that DD cannot be operable in triplet triplet energy transfer of the sort

$$
{ }^{3} D^{*}+{ }^{1} A \rightarrow{ }^{1} D+{ }^{3} A^{*}
$$

However, oscillator strengths for doublet absorption $\left(D_{\circ}\right.$ to $\left.D_{n}\right)$ can be much larger, so triplet-doublet energy transfer by the dipole mechanism may be allowed:

$$
{ }^{3} D^{*}+{ }^{2} A \rightarrow{ }^{1} D+{ }^{2} A^{*}
$$

EM energy transfer is allowed for either case.

Now we return to the simple diagram in Figure 14, but this time including spin, asking the question whether spin polarization in a triplet can be transferred to a singlet or doublet energy acceptor. In the high field limit, which is approximated in esr experiments, the triplet sublevels are $\mathrm{T}_{+}=|\alpha \alpha\rangle, \mathrm{T}_{o}=2^{-1 / 2}(|\alpha \beta\rangle+|\beta \alpha\rangle)$, and $\mathrm{T}_{-}=|\beta \beta\rangle$ and the singlet level is $2^{-1 / 2}(|\alpha \beta>-| \beta \alpha>)$ ). These levels are represented (with spin) in Figure 16.
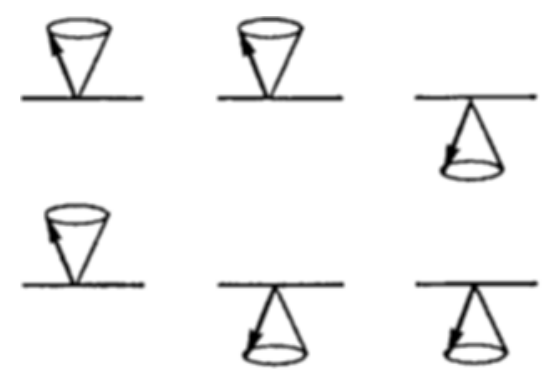

$\mathrm{T}_{+}$

$$
\mathrm{T}_{\mathrm{o}}
$$$$
\text { T. }
$$

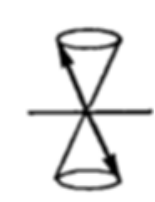

$\mathbf{S}$

Figure 16. An energy/spin vector representation of the three triplet sublevels and the singlet ground state.

Let us consider triplet doublet DD energy transfer first. Figure 17 shows that if the donor is in the $\mathrm{T}_{+}$or $\mathrm{T}_{-}$state, dipolar energy transfer cannot occur, regardless of the spin of the doublet acceptor because deactivation of the triplet would violate the Pauli principle. However, 


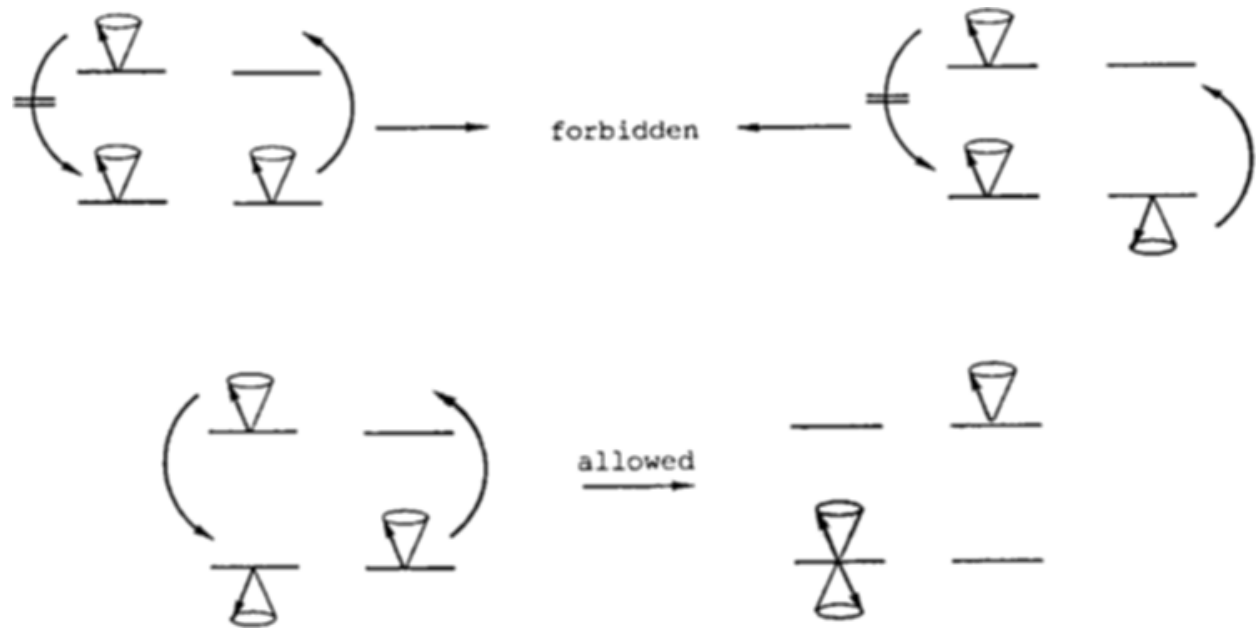

Figure 17. Dipole-dipole energy transfer for triplet-doublet systems. Regardless of the spin of the doublet, DD energy transfer is forbidden if the triplet is in the $\mathrm{T}_{+}$or $\mathrm{T}_{-}$state.

DD transfer can occur from the $\mathrm{T}_{o}$ state without restriction by the Pauli principle. But the $\mathrm{T}_{o}$ state carries no net polarization. Since all triplet mechanism polarization depends on the preferential polarization of $\mathrm{T}_{+}$or $\mathrm{T}_{-}, \mathrm{DD}$ induced energy transfer cannot contribute anything to polarization transfer!

On the other hand, Figure 18 shows that EM energy transfer can occur from a $T_{+}$state. The acceptor must contain a $\beta$ spin, which means that all singlets and half the doublets can quench. The net result of the quenching is transfer of $\alpha$ spin to the acceptor population, thus showing that EM energy transfer carries polarization transfer. With a doublet acceptor, the spin polarization transfer occurs via "plain old" spin exchange (as in section IIIE), as well. The big difference is, of course, that the doublet is left in the ground state.

Energy transfer concomitant with spin polarization transfer has been observed in a variety of systems. Akiyama, et al. showed that the direct photolysis of solutions of the dimer of 1,4-dimethylpyridyl led to $\mathrm{A} / \mathrm{E}$ RPM polarization, indicative of a singlet precursor [80,81]. Upon photolysis using benzophenone as a sensitizer, an emissive spectrum with a small A/E component was obtained at early times, suggesting there might still be some direct photolysis occurring. At slightly longer delays $(3 \mu \mathrm{s})$, the spectrum was all $E$, perhaps due to spin exchange wiping out the RPM 

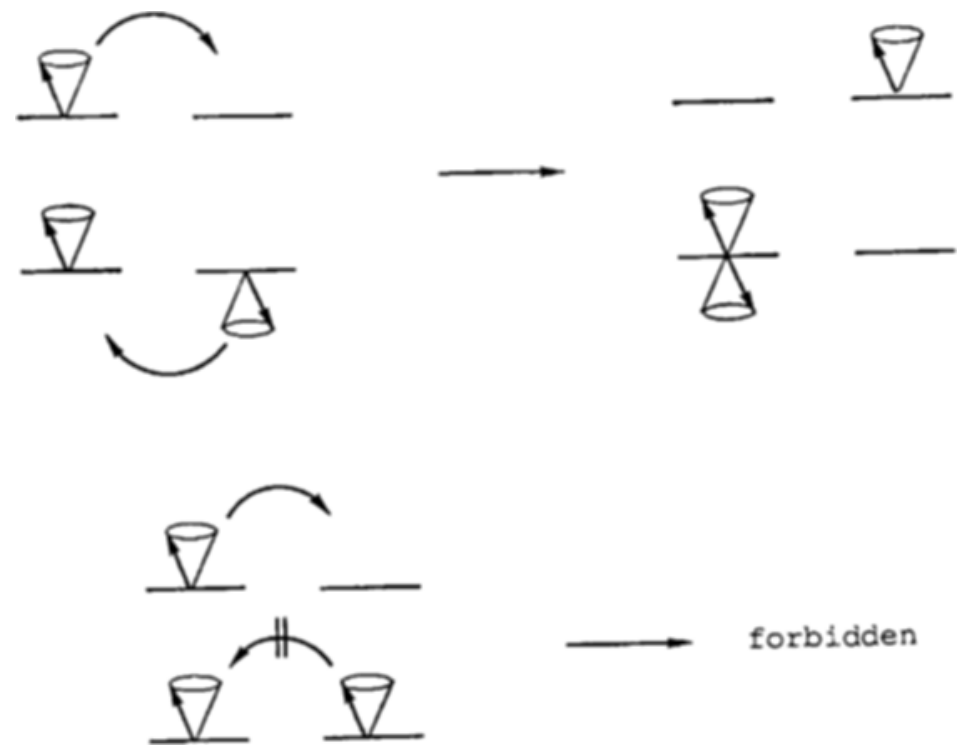

forbidden

Figure 18. Exchange mediated energy transfer for triplet-doublet systems. Energy transfer is allowed from the $\mathrm{T}_{+}$or $\mathrm{T}_{-}$level if the doublet spin is of the correct orientation. Polarization is transferred from the triplet to the doublet. See text for discussion.

polarization. Using 2-acetonaphthone as a sensitizer, an all emissive TM spectrum was obtained at all times [81].

A number of examples of triplet triplet energy transfer with conservation of spin polarization have been observed in glassy solutions at low temperature [76]. Analysis of spin polarized anisotropic triplet esr spectra allows determination of the relative populations of the sublevels. Because of the low temperature, the thermalization of the triplet sublevels is slow enough to allow direct observation. The $\Delta \mathrm{m}=2$ line of the triplet spectrum is indicative of the sign of polarization that would be observed at room temperature with standard TM polarization, since it represents the population difference between $\mathrm{T}_{+}$and $\mathrm{T}_{-}$.

Weir and Wan were the first to observe spin polarization transfer during energy transfer in low temperature randomly oriented glasses [82]. They observed the $\Delta \mathrm{m}=2$ transition of the triplet spectrum of naphthalene in the absence and presence of sensitizers. In the presence of benzophenone, which yields emissive TM, the $\Delta \mathrm{m}=2$ of naphthalene line was emissive, while in the presence of pyruvic acid, it was absorptive. Obi's group has observed similar phenomena in other systems, and 
have also observed polarization transfer in the $\Delta \mathrm{m}=1$ region, allowing modelling of the relative populations of the triplet sublevels [76].

Imamura, et al. published a study of spin polarization transfer in triplet-doublet systems [83]. They photolyzed room temperature solutions of benzophenone, phenanthrene, and pyruvic acid in the presence of the stable nitroxide, 4-hydroxy TEMPO. In the benzophenone and phenanthrene cases, they observed an emissive 3-line spectrum characteristic of the nitroxide. With pyruvic acid solutions in isopropanol, they observed the signal typical of the ketyl radical usually observed [84] (which is $\mathrm{E} / \mathrm{A}^{*}$ [85]) superimposed with a three line $\mathrm{E} / \mathrm{A}^{*}$ contribution from the nitroxide. They attributed their spectra to interaction between the nitroxide and the excited triplets in all cases, suggesting either spin exchange or EM energy transfer as the mechanism for energy transfer [76].

Nitroxides are well known quenchers of triplets, but the quenching mechanisms have yet to be fully understood. Similar compounds (e.g. fused aromatic, diphenyl polyenes, etc.) each show quenching rate profiles similar to that shown in Figure 19 as a function of triplet energy (see, for instance $[66,86,87])$. The most common assumption is that the lower plateau of the rate constant profile is due to pure spin exchange between the excited triplet and the nitroxide yielding a vibrationally excited, but electronically ground state donor and a ground state doublet and that the upper plateau is due to an energy transfer mechanism, usually thought to be EM. (No satisfactory explanation has been proposed for the rate constant minimum at $\mathrm{E}_{t}=c a .42 \mathrm{kcal} / \mathrm{mol}$.) However, detection of the excited doublet has been elusive, due to its short lifetime and lack of fluorescence, although the fluorescence of other radicals due to energy transfer has been detected [88]. Energy transfer from triplets to doublets in specific cases has been proposed to go via both DD [89] and EM $[87,90]$ mechanisms.

If we assume that the quenching of triplets by nitroxides in the upperplateau of Figure 19 is by some form of energy transfer, then the experiments of Imamura, et al. clearly demonstrate that the mechanism is EM as per the discussion in this section, or at least that EM transfer is very competitive with $\mathrm{DD}$. However, Imamura's experiments unfortunately do not prove whether any excited doublets are actually formed and thus do not distinguish spin exchange from EM. If excited doublets are formed, as seems likely, then they do show that the deactivation of the excited doublet goes by a process with spin conservation.

In this laboratory, we have observed a qualitative correlation of the intensity of a polarized nitroxide signal and the quenching rate constant, as one would expect [91]. This result does not distinguish between EM 
Quenching of Aromatic Triplets by TEMPO

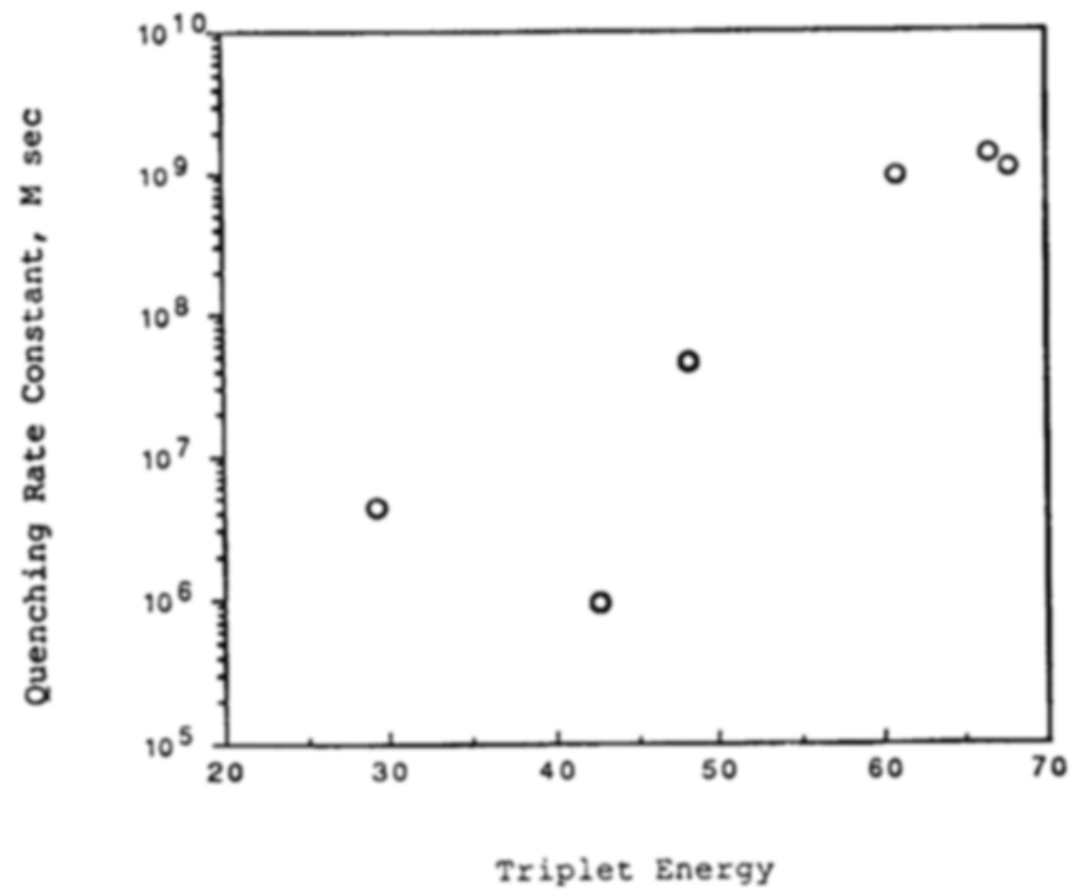

Figure 19. Rate constants for the quenching of aromatic triplets (e.g., naphthalene, phenanthrene, etc.) by TEMPO.

and spin exchange as the quenching mechanism either. We have also found (see ref. 92 and below) that it can be very tricky to decide the source of polarization in ketone/nitroxide systems when kinetic studies do not rule out either the excited triplet or the radical specifically.

Having discussed the relationship between EM energy transfer and spin exchange, it is also appropriate to discuss the relationship between EM energy transfer and electron transfer (ET). The Closs and Miller groups have studied in detail the rates of electron transfer in intramolecular cases, using molecules of the sort donor-spacer-acceptor with spacers typically being cyclohexane and decalin moieties [93]. In general, their measured rates show an exponential rate of decay with the number of bonds, demonstrating through-bond coupling, as was seen in section IIIC for biradical exchange interactions. The exponential coefficient for such decay is called $\beta$. They found that plotting points with $k_{E T}$ as the $\mathrm{x}$ axis and $k_{E M}$ as the $y$ axis for each different spacer, gave the relation that 
(within experimental error) $\beta_{E M}=2 \beta_{E T}$ [94]! More recently,they have studied positive ion, or "hole" transfer (HT) [95]. Closs, et al. proposed that for any given spacer

$$
k_{E M}=c k_{E T} k_{H T}
$$

where $c$ is the same constant for all spacers. After correcting for the charge redistribution that occurs on ET or HT, a very good correlation was observed [96].

Hole transfer (actually "back" electron transfer) can, of course, transfer polarization as well (Figure 20). Several interesting examples of this

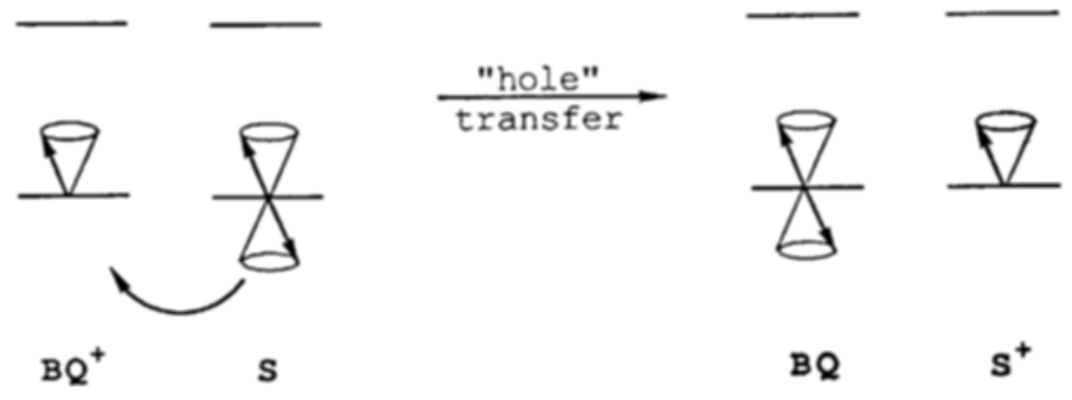

Figure 20. Polarization transfer as a result of "hole" transfer. Benzoquinone cation is produced with TM polarization, followed by electron transfer from a sulfur-containing heterocycle.

sort can be seen from the work of Wan's lab [97]. In a particular case, they found that irradiation of benzoquinone (BQ) in trifluoroacetic acid led to a TM polarized signal of $\mathrm{BQ}^{+}$due to electron ejection into solvent. Solutions containing both $\mathrm{BQ}$ and various sulfur containing compounds (RS) yielded signals attributable to both $\mathrm{BQ}^{+}$and $\mathrm{RS}^{+}$. The lack of observation of $\mathrm{BQ}^{-}$eliminated the possibility of direct electron transfer (Eq. (42)) and they were able to convincingly show that electron transfer came from the neutral sulfur compound to the polarized $\mathrm{BQ}^{+}$doublet (Eq. (43)) [98].

$$
\begin{gathered}
{ }^{3} B Q+R S \rightarrow B Q^{-}+R S^{+} \\
{ }^{3} B Q+R S \rightarrow B Q^{+}+e^{-}+R S \rightarrow B Q+R S^{+}+e^{-}
\end{gathered}
$$




\section{G. Late time phase inversion in CIDEP}

As pointed out in section IIIA, the "phase" of RPM polarization (i.e. $\mathrm{E} / \mathrm{A}$ or $\mathrm{A} / \mathrm{E}$ ) depends on the sign of $\mathrm{J}_{\text {mag res. }}$. It has almost universally been assumed that under normal circumstances, the orientationally averaged value of $\mathrm{J}$ is $>0$, i.e., that the singlet is lower than the triplet. However, there has been some speculation that this is not true in all cases.

Several reports have appeared in which radicals are initially observed in $\mathrm{E} / \mathrm{A}$ or $\mathrm{E} / \mathrm{A}^{*}$ polarization but later show $\mathrm{A} / \mathrm{E}$ or $\mathrm{A} / \mathrm{E}^{*}$ polarization [99-102]. Recalling from section IIIA that the phase of RPM polarization is dependent only the sign of $J$ and the initial spin state of the radical pair, these observations raised critical questions about the radical pair dynamics.

Carmichael and Paul, using a light modulation technique, found that geminate radical pairs formed on photolysis of di-t-butyl ketone gave $\mathrm{E} / \mathrm{A}^{*}$ polarization, but that $\mathrm{F}$-pairs exhibited $\mathrm{A}^{*} / \mathrm{E}$ signals [99]. F-pairs are thought to behave generally as triplet geminate pairs, since singlet $F$-pairs react to give diamagnetic products, leaving a triplet excess. Carmichael and Paul suggested that the RPM phase inversion was due to an inversion in the sign of $J$ for the F-pairs, due to some kind of solvent mediated exchange. They did observe a solvent dependence on the phase inversion effect, but this might be expected of almost any explanation. McLauchlan's group reported a similar effect for several aliphatic ketyl radicals generated by flash photolysis of the respective ketones [100]. They later reported some calculations which showed that if the geminate pairs and F-pairs had different trajectories (i.e. interactions began at different relative separations) and if there was a region of space where $J<0$, then triplet geminate pairs and F-pairs could give opposite RPM polarization [101].

In contrast, Thurnauer, et al. generated the same F-pairs mentioned above, using both radiolysis and hydrogen abstraction by butoxy radicals, and found that E/A polarization was universally observed by ESE techniques [103]. This and the fact that all of the previous pulse radiolysis work (which generates $\mathrm{F}$-pairs) also gave $\mathrm{E} / \mathrm{A}$ polarization seemed to rule out the explanations based on $\mathrm{J}<0$. Paul, McLauchlan, et al., however, later reported that their phase inversion observations were relatively universal, but depended strongly on radical concentration [102]. They suggested that that such a dependence could resolve the conflict between their and Thurnauer's results. They also suggested that different chemical systems (e.g. alkyl, ketyl, acyl) might inherently lead to opposite polarizations. Recent work from McLauchlan's group [104] suggests another explanation, based on a higher effective $\mathrm{J}$ for singlet radical 
pairs than for triplets, due to their ability to more closely approach each other. One might, however, expect stable radicals to produce A/E RPM polarization if this explanation were correct.

More recently, though, McLauchlan has found that all but the alkylalkyl radical pair RPM phase inversions were experimental artifacts [104]. Aware of McLauchlan's results, Muus has recently confirmed observation of phase inversion in one of the remaining cases [105]. These findings will necessitate re-examination of the experimental portions of another work by Vlyaev, Molin, Sagdeev, and the Oxford group in which electronnuclear cross-relaxation was eliminated as another proposed mechanism for the A/E polarization [106]. Jent and Paul have also very recently duplicated McLauchlan's results [107]. Their results are incompatible with the acyl/alkyl proposal and they arrive at the cross-relaxation mechanism by process of elimination. Moreover, they present calculations which suggest that, under very specific conditions of high CIDNP polarization and modulation of the hyperfine coupling at a frequency near the transition frequency (i.e., ca. $10 \mathrm{GHz}$ ), cross-relaxation via $\Delta \mathrm{m}=0$ transitions of the nuclei can lead to phase inversion. Molin and coworkers have found more support for the electron-nuclear cross-relaxation mechansim of phase inversion in spin echo experiments in the acetone-isopropanol system [107b]. The reader is referred to references [106] and [107] for details.

At this point, the "inversion phenomenon" is still a controversial question, although the cross-relaxation mechanism seems to be gaining credence. It would be worthwhile to see if the $\mathrm{A} / \mathrm{E}$ patterns seen with $t$-butyl radicals using "standard" time-resolved esr technology can be reproduced using ESE technology or fourier transfer esr at long delays (ca. $25-50 \mu \mathrm{s}$ ), even though the reported experiments (using $1 \mu \mathrm{s}$ delays) were designed to observe only F-pairs. Jent and Paul [107] suggest this should be possible with sufficiently high initial radical concentrations. Before any further speculation on the possible mechanisms of $A / E$ polarization at late times, it should be established reproducibly using different detection techniques.

\section{SPIN EXCHANGE versus REACTION: SPIN POLARIZATION TRANSFER IN DOUBLET- DOUBLET SYSTEMS}

In previous sections, spin exchange between pairs of doublets has been examined, first in its own right, then as it pertained to the development 
and quenching of RPM. In section IIIF, it was shown that an exchange interaction between a spin polarized triplet and a doublet could transfer the polarization to the doublet $[83,108]$. In this section, we demonstrate that the spin polarization of transient doublets can be transferred to persistent doublets via spin exchange.

In the slow exchange rate limit, the rate constant for spin exchange $\left(k_{e}\right)$ is given by Eq. (44)

$$
k_{e}=[R \cdot] \cdot \Delta \omega_{1 / 2} /(1-\phi)
$$

where [R.] is the concentration of the exchanging radical, $\Delta \omega_{1 / 2}$ is the observed width of the line in $\mathrm{Hz}$ less the inherent linewidth, and $\phi$ is the statistical weight of the particular hyperfine line. However, observation of line broadening by photochemically generated transient radicals in solution using this technique is often not possible; very high steady state concentrations of $c a .1 \mathrm{mM}$ would be necessary. Thus, time-resolved techniques are necessary. Bartels, et al. have shown that it is possible to measure the rate of self spin exchange using pulsed esr technology $[24,43]$, as discussed in section IIIB. We report here the use of time resolved esr and CIDEP, using only $\mathrm{CW}$ esr equipment, as an alternate probe for spin exchange between polarized transient and non-polarized persistent radicals.

Radicals formed with standard TM can transfer their polarization to a pool of unpolarized radicals by spin exchange. Such transfer is consistent with the idea that spin exchange is always faster than bond formation $[17,18,24,41]$. In the work of Bartels, et al. $[24,43]$ the rate of exchange was measured, whereas here we observe the result of the exchange.

When the transient radicals are born with an excess of a spin, then spin exchange with TEMPO tends to distribute the excess spin equally over all the radicals. Depending on the rate of exchange (i.e. polarization "equilibration"), the concentration of the transient radicals, and the relaxation rates, a TRESR spectrum would be obtained with different proportions of the transient radical and TEMPO contributions.

In order to show that spin polarization transfer was independent of chemical history of the radicals or the phase of the polarization, experiments were carried out at Columbia with chemical systems chosen to encompass three different radical generating reactions (electron transfer, hydrogen transfer, and $\alpha$-cleavage) and both absorptive and emissive TM polarizations (Please see the Appendix for experimental details.) Figure 21 shows the reaction schemes for each system.

With our system, no spectrum is observed when TEMPO alone is photolyzed in the cavity (also see reference 16). Spectra were collected 


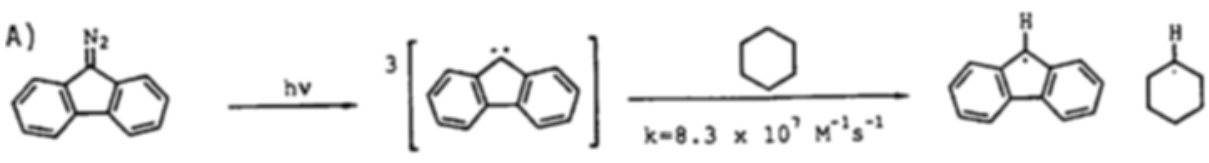

B)
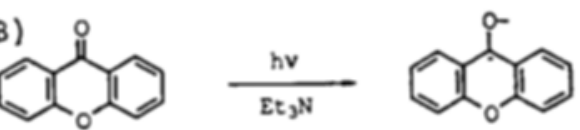

$\varepsilon t, N^{*} \longrightarrow$<smiles>OC1c2ccccc2Oc2ccccc21</smiles>

$\mathrm{Et}_{2} \mathrm{~N}-\stackrel{\mathrm{H}}{\mathrm{CH}_{3}}$

c)
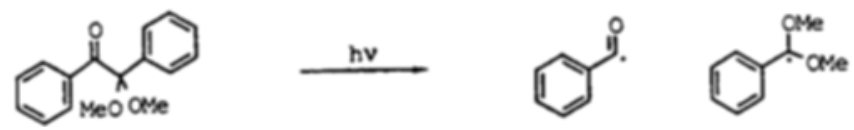

Figure 21. Reaction schemes: (a) formation of fluorenylidene, followed by hydrogen abstraction from cyclohexane; (b) photoreduction of xanthone by electron transfer from triethylamine; (c) cleavage of benzil dimethylmonoketal.

under conditions such that very little quenching interaction was expected between TEMPO and the triplet radical precursors (The actual kinetics are considered below for each case). Spectra were collected with no TEMPO and subsequently with TEMPO added to the solutions. With low concentrations of TEMPO, the observed spectra were a superposition of the original radicals and TEMPO, polarized in the same phase as the radical. It was generally found that when the TEMPO concentration was $10 \mathrm{mM}$, the observed signal was almost entirely attributable to TEMPO. Since our hypothesis was that the signal originated in the transient radical and was transferred to the persistent one, the kinetic behavior of some of the lines was investigated in some detail, as described below.

\section{A. Fluorenylidene/9-fluorenyl system: hydrogen abstraction}

The flash photolysis chemistry of fluorenylidene is by now well understood $[109,110]$. The rate constant for hydrogen abstraction by fluorenylidene from cyclohexane (Figure $21 \mathrm{a}$ ) is anomalously high for a triplet aromatic carbene at $8.3 \times 10^{7} \mathrm{M}^{-1} \mathrm{~s}^{-1}[109]$. We have taken advantage of this high rate constant and shown that the 9 -fluorenyl radical produced by photolysis of 9-diazofluorene is generated with absorptive polarization in neat cyclohexane [92]. On $266 \mathrm{~nm}$ photolysis of an argon saturated, 
A
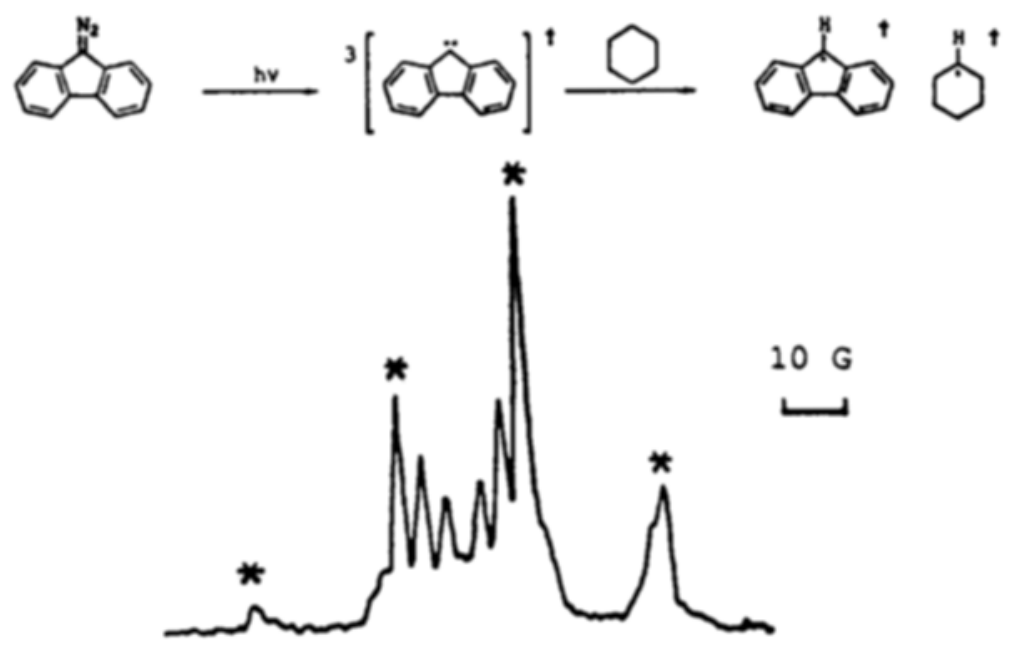

B
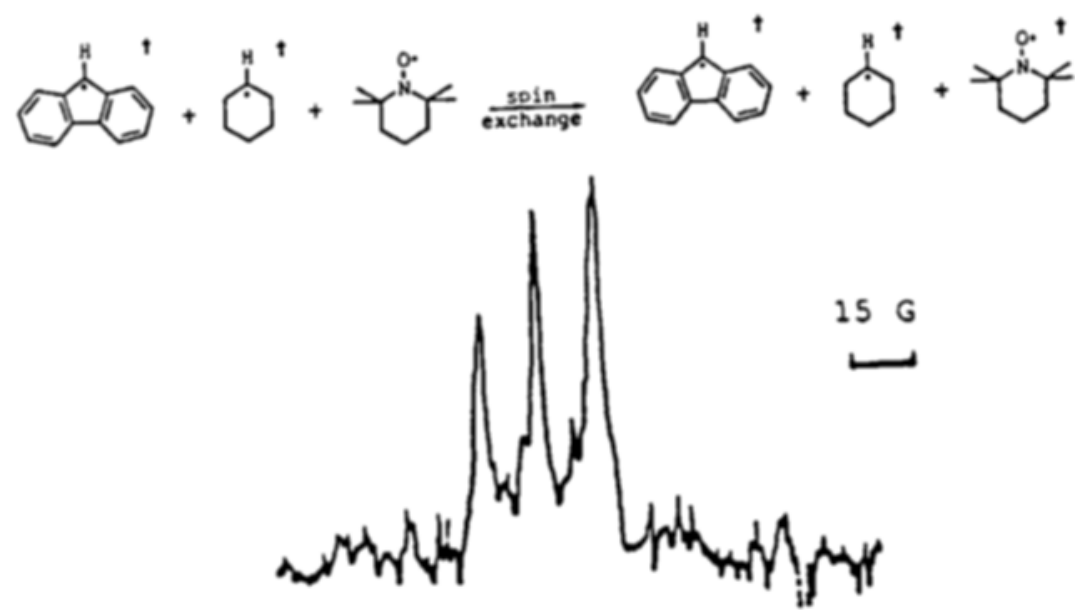

Figure 22. TRESR of fluorenyldiene system.

flowing solution of 9-diazofluorene $(5 \mathrm{mM})$ in cyclohexane the spectrum in Figure 22a is obtained with a nominal detection gate of .25 to $.5 \mu \mathrm{s}$. On addition of $10 \mathrm{mM}$ TEMPO to the solution, the spectrum in Figure 22b is obtained. No signal was obtained on photolysis of an argon saturated 5 $\mathrm{mM}$ 9-diazofluorene solution in carbon tetrachloride. Only an extremely 
weak emissive signal was obtained on photolysis of a solution of $5 \mathrm{mM}$ 9-diazofluorene and $10 \mathrm{mM}$ TEMPO in carbon tetrachloride. The signal obtained in $\mathrm{CCl}_{4}$ was essentially identical to those obtained on photolysis of solutions containing similar concentrations of fluorenone and TEMPO.

The spectrum presented in Figure 22a is characteristic of 9-fluorenyl $[92,111]$ superimposed with that of cyclohexane-1-yl with E/A* polarization. The spectrum in Figure 22b shows that spin polarization has been transferred from some transient intermediate to TEMPO. Several intermediates may be suggested, including triplet fluorenylidene, triplet fluorenone, 9-fluorenyl, and 2,2,6,6,-tetramethylaminyl radical (TMP). Since the excited diazo compound decomposes as a singlet $[109,110]$ it is not a candidate.

Triplet fluorenylidene can be eliminated on the basis of the results of photolysis in cyclohexane versus carbon tetrachloride. If spin polarization were transferred directly from triplet fluorenylidene to TEMPO, an absorptive signal would have been observed in carbon tetrachloride. The weak emissive signal actually observed is probably due to interaction between triplet fluorenone and TEMPO.

The CIDEP signals of aromatic mono-carbonyls probed by either photoreduction [3] or interaction with TEMPO [83] are emissive. We have found similar results for fluorenone under a variety of conditions, even though its lowest triplet is not an $n, \pi^{*}$ state. The signals obtained with fluorenone are always substantially weaker than those from $n, \pi^{*}$ ketones. In any case, the signals obtained here are absorptive, so triplet fluorenone is eliminated as the source of polarization for TEMPO.

Having eliminated the triplets as spin polarization sources, the two possible radical sources are considered. In neat cyclohexane, the rise time of 9-fluorenyl is $1.4 \mathrm{~ns}$ [109]. Diphenylmethylene reacts with TEMPO to produce benzophenone and TMP with a rate constant of $7.3 \times 10^{8}$ $\mathrm{M}^{-1} \mathrm{~s}^{-1}$ [112]. The rate constant for analogous reactive quenching of fluorenylidene (in perfluorobenzene) by TEMPO was found to be $3.1 \pm .5$ $\times 10^{8} \mathrm{M}^{-1} \mathrm{~s}^{-1}$. Thus, with a TEMPO concentration of $10^{-2} \mathrm{M}$, up to a few percent of the fluorenylidene may react with TEMPO to generate fluorenone and TMP. Infrared analysis of the solutions used in generating spectra like $22 \mathrm{~b}$ suggests that some fluorenone is present after photolysis. TMP could be independently generated by net hydrogen abstraction by either triplet benzophenone or $t$-butoxy radicals from tetramethylpiperidine in time-resolved and steady state experiments, respectively. By admission of oxygen to the system, TEMPO and TMP could be observed simultaneously. The ${ }^{14} \mathrm{~N}$ coupling constants of TEMPO and TMP (14.5 and 15.5 
$\mathrm{G}$, respectively, depending on the solvent) allow distinction between their respective esr signals; the signal in Figure $22 \mathrm{~b}$ is from TEMPO.

It seems clear that the source of the spin polarization transferred to TEMPO is 9-fluorenyl, with TMP possibly contributing. Thus the polarization starts in triplet fluorenylidene (unobserved), is preserved in the hydrogen abstraction reaction to form 9-fluorenyl (observed in the absence of TEMPO), and is transferred by spin exchange to TEMPO. By time resolved laser flash spectroscopy, we found that TEMPO reactively quenched 9-fluorenyl with a rate constant of $8.3 \pm .4 \times 10^{7} \mathrm{M}^{-1} \mathrm{~s}^{-1}$ in cyclohexane. This compares with rates of $5.5 \pm .5 \times 10^{8} \mathrm{M}^{-1} \mathrm{~s}^{-1}$ and $4.63 \pm .02 \times 10^{7} \mathrm{M}^{-1} \mathrm{~s}^{-1}$ for benzyl and diphenylmethyl, respectively, in isooctane [113], and is approximately two orders of magnitude slower than diffusion controlled. As it is expected that the spin exchange rate constant between the radicals in this study and TEMPO is essentially half the diffusion controlled rate [28], there is clearly ample opportunity for multiple collisions (and thus spin exchange) between the radicals before they are destroyed by reaction.

\section{B. Xanthone system: electron transfer}

Triethylamine is an efficient electron transfer donor for excited triplet ketones $[114,115]$. Flash photolysis experiments showed that triplet xanthone (monitored by triplet-triplet absorption at $610 \mathrm{~nm}$ ) was quenched by triethylamine (Figure $21 \mathrm{~b}$ ) with a rate constant of $1.09 \pm .02 \times 10^{10}$ $\mathrm{M}^{-1} \mathrm{~s}^{-1}$ in acetonitrile at room temperature. TEMPO was found to quench xanthone triplets with a rate constant of $8.2 \pm .3 \times 10^{9} \mathrm{M}^{-1} \mathrm{~s}^{-1}$. Thus triplet xanthone quenching by TEMPO is not competitive with photoreduction at TEMPO concentrations of $10 \mathrm{mM}$ or less in neat triethylamine.

Sakaguchi and Hayashi have reported both absorptive and emissive xanthone ketyl CIDEP signals under different conditions, using organometal hydrides as reducing agents $[114,115]$. In our hands, reduction of xanthone triplet with triethylamine in a variety of solvents always leads to an emissive signal.

Time resolved esr spectra were collected for $5 \mathrm{mM}$ solutions of xanthone in neat triethylamine with $355 \mathrm{~nm}$ excitation. They are shown in Figures 23a-d with TEMPO concentrations of $0,1,5$, and $10 \mathrm{mM}$. The emissive spectra show the overlap of signals due to the reduction product of xanthone [114,115], 1-N,N-diethylaminoeth-1-yl [116], and TEMPO. Again, $10 \mathrm{mM}$ TEMPO was sufficient to dominate the signal. A solution of xanthone $(5 \mathrm{mM})$ and TEMPO $(10 \mathrm{mM})$ in methylene chloride without 

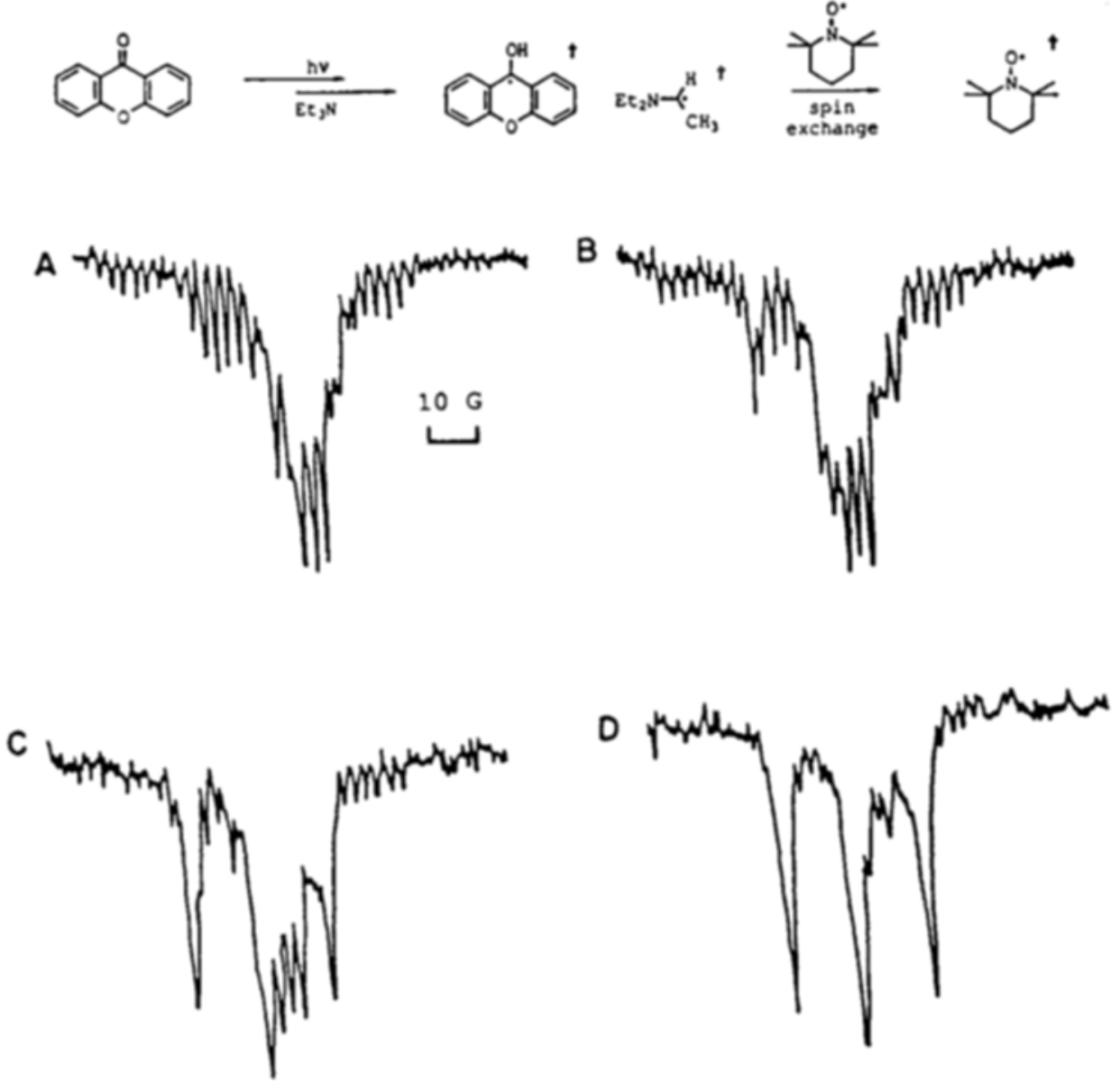

Figure 23. TRESR of xanthone system. The spectra were obtained from solutions of $5 \mathrm{mM}$ xanthone in neat triethylamine with (a) $0 \mathrm{mM}$, (b) 1 $\mathrm{mM}$, (c) $5 \mathrm{mM}$, (d) $10 \mathrm{mM}$ TEMPO.

any added reducing agent produced very little or no signal. These weak signals may be due to inefficient direct interaction between the triplet and TEMPO or interaction between the ketyls formed by inefficient hydrogen abstraction from solvent and TEMPO.

Under the conditions of our experiments, the maximum rate of interaction between TEMPO and triplet xanthone (ca. $10^{8} \mathrm{~s}^{-1}$ ) is three orders of magnitude slower than the rate of photoreduction of the triplet (ca. $10^{11} \mathrm{~s}^{-1}$ ). The source of spin polarization for TEMPO must then be the xanthone radical anion or the subsequently formed ketyl. 
The ratio of signal due to TEMPO and xanthone radical as a function of TEMPO concentration is a complex function of many factors, including the spin exchange rate, the inherent electronic $T_{1}$ and $T_{2}$ of the ketyl, the triethylamine radical, and TEMPO, and the relaxation induced by the presence of the microwave field. We thus do not try to quantify the signals here, but note that the non-TEMPO signal strength maxima for solutions containing $0,1,5$, and $10 \mathrm{mM}$ TEMPO were about $100,56,39$, and 13 , respectively (arbitrary units).

\section{Benzil dimethyl monoketal (BDM) system: $\alpha$-cleavage}

$\mathrm{BDM}$ is an efficient radical polymerization initiator. On photolysis, a benzoyl radical and a dimethoxybenzyl radical are formed (Figure 21c). The spectra we obtained on photolysis of BDM in the absence of TEMPO are in agreement with the published results, although we are not able to achieve the same high resolution with our equipment [117]. The results of adding TEMPO to the solution are essentially identical to the xanthone system, suggesting that the qualitative relaxation and spin exchange rates are similar in the two systems. A spectrum obtained from a $10 \mathrm{mM}$ solution of BDM in methylene chloride with a $1-2 \mu$ s gate and $355 \mathrm{~nm}$ excitation is shown in Figure 2. The majority of the signal is due to the dimethoxybenzyl radical. The benzoyl radical, which has a substantially shorter relaxation time than the dimethoxybenzyl (DMB) radical, is not observed in this time window. In Figure 24 are four spectra obtained with gates of nominally $400-900$ ns with TEMPO concentrations of $0,1,5$, and $10 \mathrm{mM}$.

The BDM system was chosen to examine the temporal behavior of the transient and stable radicals. It was expected that the grow-in of the DMB polarization should be instrument limited, while the grow-in of TEMPO spin polarization should be dependent on the overall rate of spin exchange, which could be adjusted by changing the TEMPO concentration and the viscosity of the solvent.

Figure 25a shows the kinetic behavior of the low field TEMPO line and the center of the DMB spectrum taken in dodecane solvent. The time of the peak of the DMB signal was shown to be approximately constant over the range of TEMPO concentrations used as shown in Figure $25 \mathrm{~b}$, although the apparent decay rate is faster for the sample with more TEMPO, as expected. Figure 25c compares the behavior of the low field TEMPO peak in two solvents, isooctane and decalins, while Figure 25d 

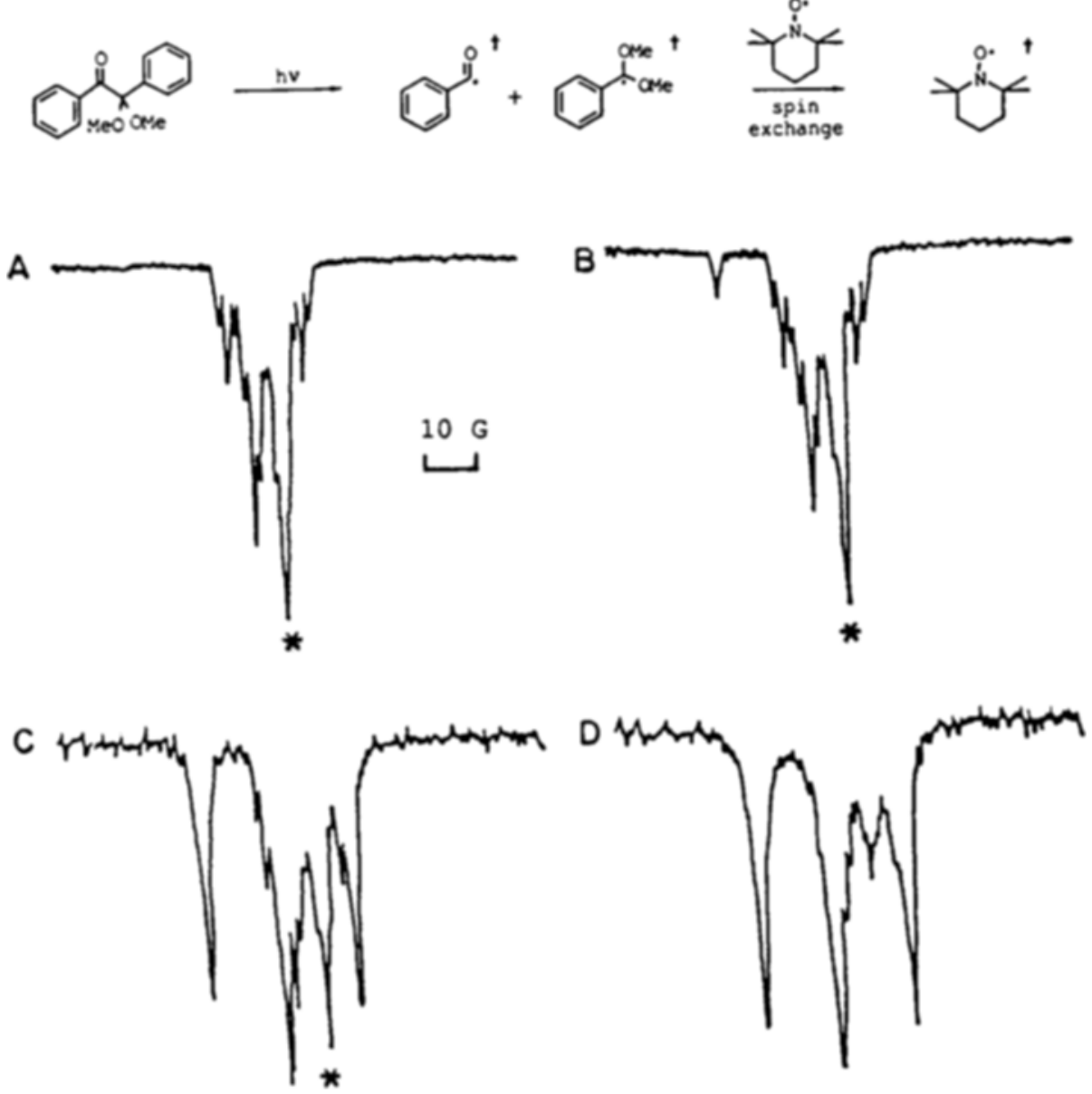

Figure 24. TRESR of the benzil dimethyl monoketal system. The spectra were obtained from solutions with (a) $0 \mathrm{mM}$, (b) $1 \mathrm{mM}$, (c) $5 \mathrm{mM}$, (d) 10 $m M$ TEMPO. The benzoyl radical appears only as additional intensity in the peak marked with a star.

compares the same peak's behavior as a function of TEMPO concentration in dodecane.

Based on the assumption that the grow in of the DMB signal is instrument-limited, the apparent response time of our TRESR system is between 150 and $250 \mathrm{~ns}$. Because we use the actual laser pulse for the timing of the boxcar (to minimize trigger jitter), some delay is introduced and we are unable to see the true time $=0$ point or measure the response function precisely. As relaxation begins, there is obviously some point at 

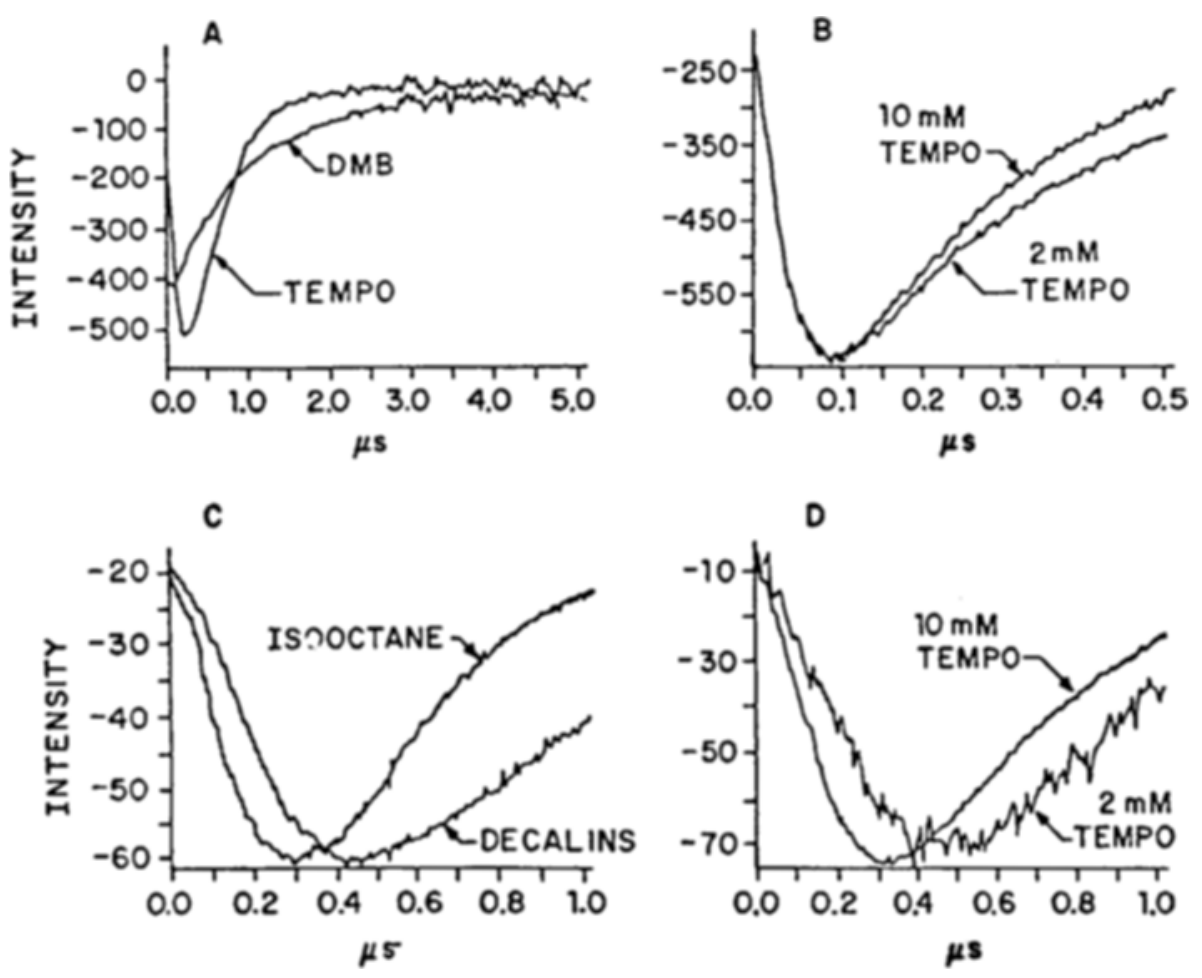

Figure 25. Time profiles of key peaks: (a) dimethoxy benzyl (DMB) and TEMPO (10 $\mathrm{mM}$ ) in cyclohexane; (b) dimethoxy benzyl peak with $2 \mathrm{mM}$ and $10 \mathrm{mM}$ TEMPO; (c) TEMPO peak (10 mM) in isooctane and decalins; (d) TEMPO peak, $2 \mathrm{mM}$ and $10 \mathrm{mM}$. See text for discussion.

which the signal is maximized $\left(t_{\max }\right)$, with faster relaxation leading to an earlier signal maximum. Slower relaxation leads to a later, broader $t_{\max }$. Alternatively, the slow growth of a signal, followed by relatively fast relaxation also gives a later $t_{m a x}$, but is easily distinguishable from the former case.

In Figure 25a, it is clear that the $t_{\max }$ of the TEMPO line comes later, despite its faster relaxation rate. This implies that TEMPO is acquiring signal intensity on a timescale substantially longer than the laser pulse width. We suggest that TEMPO has essentially zero spin polarization at $t=0$ and gains intensity continuously from DMB through the spin exchange process.

We can adjust the spin exchange rate by changing the TEMPO concentration. As long as the TEMPO concentration remains low enough that self-exchange remains in the slow exchange limit, a larger TEMPO 
concentration should lead to an earlier $t_{\max }$. This prediction is confirmed in Figure 25d, which shows $t_{\max }$ of about $500 \mathrm{~ns}$ with $2 \mathrm{mM}$ TEMPO and 300 ns with $10 \mathrm{mM}$ TEMPO.

Secondly, since $\mathrm{k}_{e x} \sim 0.5 \mathrm{k}_{d i f f}$ and $\mathrm{k}_{d i f f}$ is inversely proportional to viscosity, it is expected that $t_{\max }$ should shift to later times with higher viscosity. This prediction is confirmed in Figure 25c, which shows that solutions with $10 \mathrm{mM}$ TEMPO in isooctane and decalins have $t_{\max }$ about $300 \mathrm{~ns}$ and $400 \mathrm{~ns}$, respectively. Because some heating of the samples due to the repetitive laser flashes is inevitable, the temperatures of the samples are not precisely known. The room temperature viscosities of isooctane and mixed decalins are 5.03 and $24.2 \mathrm{cP}$, and because the sample heating is not more than a few percent, it is expected that the actual viscosities are similar.

Also consistent with the viscosity predictions is the result that the difference in $t_{\max }$ between solutions of 2 and $10 \mathrm{mM}$ TEMPO also increases with viscosity, being approximately $100 \mathrm{~ns}$ in isooctane and 200 ns in mixed decalins. We believe that this result argues against the viscosity effect being due only to changes in TEMPO $\mathrm{T}_{1}$ because of increased rotational correlation time.

Because of general intensity differences in the BDM/TEMPO signals in different solvents, the viscosity dependence of the BDM signal (alone) was checked. Spectra were obtained from solutions of $10 \mathrm{mM}$ BDM in hydrocarbon solvents. A general increase in signal intensity with viscosity was observed. As measured by the signal intensity at $t_{\max }$, the signal intensity varied a factor of about 3.5 over the solvents n-pentane $(\eta=$ $2.15 \mathrm{cP}$ at room temperature) through mixed decalins $(\eta=24.2 \mathrm{cP})$.

This effect is probably attributable to an increase in rotational correlation time [1]. The signal increase arises because intersystem crossing occurs faster $\left(10^{-11}-10^{-12} \mathrm{~s}\right)$ than the triplet sublevels can respond to the magnetic field. Thus the polarization is born in the molecular frame. The sublevels respond to the magnetic field in the laboratory axis at the inverse of the Zeeman frequency,

$$
\left(\omega_{z}\right)^{-1}=(g \beta H / h)^{-1} \approx 1.7 \times 10^{-11} s
$$

However, if the rotational correlation time is fast in comparison to the Zeeman response time, the correlation between the molecular and laboratory frames is lost and polarization is diminished or lost entirely. Although we have no direct measurement of the correlation time of our intermediates, increasing viscosity of solvent certainly causes an increase in correlation time. This data is not of sufficient quantity or quality to try to fit to a specific function. 
After the conclusion of this work, we ran across a reference to similar work [118] that seems to have preceded both ours and Imamura, et al. [83]. In this preliminary work, the authors used pulsed esr technology to show spin polarization transfer from triplet duroquinone and what was probably a mixture of triplet duroquinone (DQ) and its radical anion to 3-phenyl-2,2,5,5-tetramethylimidizoline-1-oxyl (RNO) and proposed that such a technique could be used to study short-lived radicals. They found a maximum in the spin-echo signal corresponding to about 0.6 and $0.9 \mu \mathrm{s}$ for solutions that contained $\mathrm{DQ}^{-}$and $5 \times 10^{-4} \mathrm{M}$ and $2 \times 10^{-4} \mathrm{M}$ RNO, respectively. This work is clearly in qualitative agreement with both the present results and those of Imamura.

It should be pointed out that reaction between TEMPO and polarized radicals will lead to net polarization of TEMPO by depletion of TEMPO molecules of the opposite spin state from that favored by the polarized molecule. Rreaction between TEMPO and a polarized radical transfers one "unit" of magnetization from the polarized radical to TEMPO, whereas exchange transfers two because the loss of the depleted spin state is coupled to the gain of a spin in the polarization-favored state. Thus the contribution of this mechamism to the growth of polarization, relative to spin exchange should be given the fraction $\mathrm{k}_{r \times n} /\left(\mathrm{k}_{r x n}+2 \mathrm{k}_{e x}\right)$.

For the example of fluorenylidene, the "reaction" contribution is less than $1 \%$ if $\mathrm{k}_{e x}$ is taken as $0.5 \mathrm{k}_{\text {diff }}$. The contribution from more reactive readicals (such as cycloxhexane-1-yl) will be larger. Reaction rate constants between even primary radicals and TEMPO in isooctane are about $1 \times 10^{9} \mathrm{M}^{-1} \mathrm{~s}^{-1}$, which leads to a reactio contribution of $<10 \%$. Bordat and Tsvetkov found $\left(k_{e x}+k_{r x n}\right)=6.6 \times 10^{9} \mathrm{M}^{-1} \mathrm{~s}^{-1}$ for $\mathrm{DQ}^{-}$and RNO [119], which is clearly too large to be anything but dominated by the $k_{e x}$ term. Clearly, these two mechanisms are coupled, and the relative contributions depend on the reactivity of the radical pair. However, the results discussed in section IIIG demand that $\mathrm{k}_{e x}>\mathrm{k}_{r x n}$; Bartels, et al. show the exchange radius for even the ethanol-1-yl radical. In the limit where $k_{e x}=k_{r x n}$, the reaction contribution maximizes at $33 \%$.

With regard to the study of very reactive radicals by this method, cautious optimism is due. Good kinetic analysis of such systems will be complex due to the addition of the passive CIDEP effect [5], which becomes important when the reaction rate of the radicals becomes similar to their relaxation rate. In order to capture very fast decaying polarizations, rather high nitroxide concentrations may be necessary. In addition to capturing signal, such concentrations will certainly decrease relaxation times as observed in our work and optical windows may be hard to find. Moreover, dipole/dipole interactions may become important at the high 
concentrations and these short lived radicals will have to be generated with very large net polarizations, which may be problematic.

In this section, we have shown that spin-polarized transient radicals can be generated under conditions such that their polarization is transferred to an originally unpolarized pool of persistent radicals. The precursors to the radicals are not involved in polarization transfer in the examples given here and exchange occurs with either radical polarization. The radical generating reaction is not important, so long as polarization is established. Because of limitations of our instrumentation, we have not been able to be rigorous in demonstrating kinetics, but have shown that the polarization behaves qualitatively as expected.

The mechanism of polarization transfer must be extremely fast to compete favorably with radical-radical reaction. Dipole-dipole interactions are not important at these concentrations [15] and would be expected to be averaged out by isotropic tumbling. Since the rate of spin exchange is always faster than the rate of reaction [17] as shown for even diffusion controlled reactions by Bartels, et al., we conclude that the polarization transfer mechanism is spin exchange.

\section{SUMMARY}

We have presented a review of a number of different electron exchange interactions, which range from Heisenberg spin exchange to energy transfer. We have chosen to present most of this material largely from the perspective of esr experiments, but that is an arbitrary choice. One could build experiments around energy transfer or $\mathrm{nmr}$ experiments (or several other things!) and make nearly the same points. It is hoped that the reader will come away with the idea that all these exchange phenomena are related to each other, and that often the differences between their manifestations are usually remnants of the order of magnitude of the detailed observation, rather than fundamental differences.

\section{APPENDIX: EXPERIMENTAL SECTION}

\section{Materials}

All solvents used were of the highest quality commercially available, typically "spectro" or "HPLC" grade, and were used without further purification, except as noted. Triethylamine was distilled prior to use. TEMPO was purchased from Aldrich and used as received. Xanthone was from Aldrich and multiply recrystallized from ethanol. Benzil dimethyl 
monoketal (BDM) was a kind gift of the Ciba-Geigy Corporation (Irgacure 651). Diazofluorene was prepared by literature methods [119].

\section{Time-Resolved ESR measurements}

The apparatus used has been described before and consists of a Bruker ER 100D ESR with a broad band $(6.6 \mathrm{MHz})$ preamplifier, a Quantel Nd:Yag laser and an EG\&G PARC boxcar model 4400 with model 4421 integrators, yielding a response time of $c a .150-250 \mathrm{~ns}$ [120].

Argon flushed solutions were flowed through a flat cell $.5 \mathrm{~mm}$ deep in the cavity at a rate of $1-5 \mathrm{ml} / \mathrm{min}$. Excitation was provided by the laser (ca. $15 \mathrm{~ns}, 20 \mathrm{~Hz}$ ) operating at $266 \mathrm{~nm}(5-10 \mathrm{~mJ} /$ pulse) or 355 $\mathrm{nm}(25-50 \mathrm{~mJ} /$ pulse). The directly detected signal was sent via a broad band amplifier to the boxcar for either spectra or kinetic traces. Most spectra were collected over $500 \mathrm{~s}$ with the boxcar gates open from .5 to $1 \mu \mathrm{s}$ after each laser shot. The instrument was calibrated for magnetic field and absorptive/emissive phase using the well known $\mathrm{E} / \mathrm{A}^{*}$ pattern of the acetone/isopropanol system [121] and was time-calibrated using a multi-channel delay box.

For spectra, the magnetic field was slowly swept as the boxcar collected points. For kinetic measurements, an off resonance kinetic trace was subtracted from each on resonance trace to correct for thermal and other effects in the cavity. All measurements were made with (nominally) $1.8 \mathrm{~mW}$ microwave power. This level was found to be sufficient to provide strong signals without causing Torrey oscillations. The kinetic traces were found to decay only slightly faster than at .18 or $.018 \mathrm{~mW}$ and the signal-to-noise ratio was substantially better.

\section{Flash photolysis}

The apparatus used for the flash studies has been described before [122]. Excitation was provided by either a Lambda Physik EMG 201 laser operating at $308 \mathrm{~nm}$ or by a Quantel DCR $2 \mathrm{Nd}$ :Yag operating at $355 \mathrm{~nm}$. Solutions had optical densities of .3 to .5 at the excitation wavelength and were deoxygenated by argon flushing. Radicals, triplets, and carbenes were all monitored by transient absorption at literature reported wavelengths. When triethylamine was used, solutions were bubbled for 4 minutes at $0{ }^{\circ} \mathrm{C}$ then warmed to room temperature before measurement. All rate constants were fitted to the equation $\mathrm{k}_{o b s}=\mathrm{k}_{\text {inherent }}+\mathrm{k}_{q}[\mathrm{Q}]$ with at least 5 concentrations and at least 3 points per concentration, except for the reaction of fluorenylidene and TEMPO, for which there 
were 4 concentrations with at least 5 points per concentration. Reported errors are 2 standard deviations of the slope obtained from the fit.

\section{ACKNOWLEDGEMENTS}

The authors thank the the NSF, the AFOSR, and the DOE for their generous support of this work. W.S.J. thanks the NSF for a predoctoral fellowship and the Division of Organic Chemistry of the American Chemical Society sponsored by the Monsanto Company for support during a portion of this work. The authors are grateful to Professor Matthew Platz for a preprint of his review of carbene chemistry, to Professor K.A. McLauchlan for preprints of his CIDEP reviews, and to Dr. C.D. Doubleday, Jr. and Dr. Valery Tarasov for stimulating discussions.

\section{REFERENCES}

1. For two recent reviews of CIDEP see (a) F.J. Adrian, Rev. Chem. Int., 7(1986) 173; (b) K.A. McLauchlan and D.G. Stephens, Acc. Chem. Res., 21(1988)54; see also (c) A.D. Trifunac, R.G. Lawler, D.M. Bartels, and M.C. Thurnauer, Prog. in Reaction Kinetics, 14(1986)43; A substantial amount of work on photosynthetic systems has been done, which will not be discussed here. A review of this work can be found in (d) A.J. Hoff, Q. Rev. Biophys., 17(1984)153, and in (c).

2. Adapted from ref. 1 (a).

3. (a) A.I. Grant and K.A. McLauchlan, Chem. Phys. Lett., 101(1983)120; (b) S. Yamauchi and N. Hirota, J. Phys. Chem., 88(1984)4631.

4. (a) J.K.S. Wan and A.J. Elliot, Acc. Chem. Res., 10(1977)161; (b) F.J. Adrian and L. Monchick, J. Chem. Phys., 71(1979)2600; (c) F.J. Adrian and L. Monchick, J. Chem. Phys., 72(1980)5786.

5. (a) D.M. Bartels and R.G. Lawler, J. Chem. Phys., 86(1987)4843; (b) D.M. Bartels, J. Mag. Res., 76(1988)341.

6. (a) R.W. Fessenden and R.H. Schuler, J. Chem. Phys., 39(1963)2147; (b) P. Neta, R.W. Fessenden, and R.H. Schuler, J. Phys. Chem., 75(1971)1654; (c) N.C. Verma and R.W. Fessenden, J. Chem. Phys., 58(1973)2501; (d) R.W. Fessenden and N.C. Verma, Discuss. Faraday Soc., 63(1977)104.

7. (a) D. Beckert and K. Mehler, Ber. Busenges. Phys. Chem., 88(1984)1013; (b) D.M. Bartels, T.M. Chiu, A.D. Trifunac, and R.G. Lawler, Chem. Phys. Lett., 123(1986)497.

8. (a) M. Anpo, R. Sutcliffe, and K.U. Ingold, J. Am. Chem. Soc., 105(1983) 3580; (b) M. Anpo, K.U. Ingold, and J.K.S. Wan, J. Phys. Chem., 87(1983) 1674; (c) T.J. Burkey, J. Lusztyk, K.U. Ingold, J.K.S. Wan, and F.J. 
Adrian, J. Phys. Chem., 89(1985)4286; (d) C.D. Buckley and K.A. McLauchlan, Chem. Phys. Lett., 137(1987)86; (e) F.J. Adrian, K. Akiyama, K.U. Ingold, and J.K.S. Wan, Chem. Phys. Lett., 155(1989) 333.

9. (a) A.D. Trifunac, Chem. Phys. Lett., 49(1977)457; (b) A.D. Trifunac, D.J. Nelson, and C. Mottley, J. Mag. Res., 30(1978)263.

10. L. Salem and C. Rowland, Angew. Chem., Int. Ed. Engl., 2(1972)92.

11. V.N. Parmon, A.I. Kokorin, and G.M. Zhidomirov, J. Struct. Chem., 18(1977)104, translated from Zh. Strukt. Khim., 18(1977)133.

12. C. Doubleday, Jr., J.W. McIver, Jr., and M. Page, J. Am. Chem. Soc., $104(1982) 6533$.

13. A.H. Goldberg and D.A. Dougherty, J. Am. Chem. Soc., 105(1983)284.

14. F.W. Bromowicz and W.A. Goddard, III, in H.F. Schaefer, III (Ed.), Methods of Electronic Structure Theory, Plenum Press, New York, 1977.

15. Y.N. Molin, K.M. Salikhov, and K.I. Zamaraev, Spin Exchange, Springer Verlag, New York, 1980.

16. This is the form of the Hamiltonian often used in the spin exchange literature. The spin Hamiltonian is perhaps more properly written as $\mathrm{J}(1 / 2$ $+2 S_{1} \cdot S_{2}$ ). This latter form is used by many authors. Both forms lead to the same results for all the calculations done here, so we will use the simpler form consistently.

17. (a) J.A. Syage, J. Chem. Phys., 87(1987)1022; (b) J.A. Syage, J. Chem. Phys., 87(1987)1033.

18. F.J. Adrian, J. Chem. Phys., 88(1988)3216.

19. F.J. Adrian, J. Chem. Phys., 57(1972)5107.

20. T.J. Burkey, J. Lusztyk, K. U. Ingold, J.K.S. Wan, and F.J. Adrian, Phys. Chem., 89(1985) 4286.

21. The method used in ref. 23 is due to P.V. Schastnev and K.M. Salikhov, Theor. Exper. Chem., 9(1975)223, translated from Teor. Eksp. Khim., $9(1975) 291$.

22. R.N. Musin and P.V. Schastnev, J. Struct. Chem., 17(1976)362, translated from Zh. Strukt. Khim., 17(1976)419.

23. (a) J.D. Currin, Phys. Rev., 126(1962)1995; (b) C.S. Johnson, Mol. Phys., $12(1967) 25$.

24. D.M. Bartels, A.D. Trifunac, and R.G. Lawler, Chem. Phys. Lett., 152(1988) 109.

25. H. Shimizu, J. Chem. Phys., 42(1965)3599.

26. J.A. Syage, personal communication.

27. See, for instance, T.A. Miller and R.N. Adams, J. Am. Chem. Soc., 88(1966)5713.

28. Reference 15, chapter 4.

29. J.H. Freed, J. Chem. Phys, 71(1967)38. 
30. (a) P.J. Hore and K.A. McLauchlan, Mol. Phys., 42(1981)533; (b) S. Basu, K.A. McLauchlan, and A.J.D. Ritchie, Chem Phys. Lett., 105(1984)447; (c) K.A. McLauchlan and A.J.D. Ritchie, Mol. Phys., 56(1985)141; (d) K.A. McLauchlan and A.J.D. Ritchie, Mol. Phys., 56(1985)1357; (e) K.A. McLauchlan and D.G. Stevens, J. Chem. Phys., 87(1987)4399.

31. F.J. Adrian, J. Chem. Phys., 54(1971)3918.

32. R.M. Noyes, J. Chem. Phys., 22(1954)1349.

33. a) J.B. Pedersen and J.H. Freed, J. Chem. Phys., 58(1973)2869; (b) J.B. Pedersen and J.H. Freed, J. Chem. Phys., 59(1973) 2746.

34. Pedersen and Freed's work (including reference 30 ) is presented as a review in Adv. Mag. Res., 8(1976)1.

35. A similar treatment can be found in K.A. McLauchlan in Application of Lasers in Polymer Science Technology, J.P. Fouassier and J.F. Rabek, CRC Press, 1989.

36. L. Monchick and F.J. Adrian, J. Chem. Phys., 68(1978)4376.

37. G.P. Zientara and J.H. Freed, J. Phys. Chem., 83(1979)3333.

38. F.J. Adrian and L. Monchick, J. Chem. Phys., 71(1979)2600.

39. F.J. Adrian, J. Chem. Phys., 88(1988)3216.

40. K.A. McLaughlan and D.G. Stephens, J. Chem. Phys., 87(1987)4399.

41. J.A. Syage, Chem. Phys. Lett., 108(1984)266.

42. P.P. Bordat and Y.D. Tsvetkov, Doklady Phys. Chem., 278(1985)865, translated from Doklady Adademii Nauk SSSR, 4(1984)900.

43. J.A. Syage, R.G. Lawler, and A.D. Trifunac, J. Chem. Phys., 77(1982)4774.

44. H. Paul, Chem. Phys., 40(1979)265.

45. J.E. Wertz and J.R. Bolton, Electron Spin Resonance: Elementary Theory and Practical Applications, McGraw-Hill, New York, 1972, pp. 251 ff.

46. G.R. Eaton and S.S. Eaton, Acc. Chem. Res., 21(1988)107.

47. Y. Sakaguchi, H. Hayashi, H. Murai, and Y.J. I'Haya, Chem. Phys, Lett., $110(1984) 275$.

48. (a) K.A. McLauchlan and D.G. Stephens, Chem. Phys. Lett., 115(1985)108;

(b) H. Hayashi, Y. Sakaguchi, H. Murai, and Y.J. I'Haya, Chem. Phys. Lett., 115(1985)111; (c) Y. Sakaguchi, H. Hayashi, H. Murai, Y.J. I'Haya, and K. Mochida, Chem. Phys. Lett., 120(1985)401; (d) H. Murai, Y. Sakaguchi, H. Hayashi, and Y. J. I'Haya, J. Phys. Chem., 90(1986)113.

49. A.D. Trifunac and D.J. Nelson, Chem. Phys. Lett., 46(1977)346.

50. C.D. Buckley, D.A. Hunter, P.J. Hore, and K.A. McLauchlan, Chem. Phys. Lett., 135(1987)307.

51. G.L. Closs, M.D.E. Forbes, and J.R. Norris, J. Phys. Chem., 91(1987)3592.

52. G.L. Closs, and M.D.E. Forbes, J. Am. Chem. Soc,, 109(1987)6185.

53. References 51-53 use 2J for the $S-T_{o}$ splitting. This is because they use $J\left(1 / 2+2 S_{1} \cdot S_{2}\right)$ for the spin exchange Hamiltonian, rather than $J_{1}$ 
- $\mathbf{S}_{2}$, as we have been using. The correspondence is straightforward. See reference 16.

54. N.J. Turro, M.B. Zimmt, and I.R. Gould, J. Am. Chem. Soc., 105(1983)6347.

55. G.L. Closs, personal communication.

56. J.F. Wang, C.D. Doubleday, Jr., and N.J. Turro, J. Am. Chem. Soc., $111(1989) 3962$.

57. M.C. Thurnauer and J.R. Norrik, Chem. Phys. Lett., 76(1980)557.

58. (a) M.K. Bowman, D.E. Budil, G.L. Closs, V.A.G. Kostka, C.A. Wraight, and J.R. Norris, Proc. Nat. Acad. Sci., 78(1981)3305; (b) J.R. Norris, M.K. Bowman, D.E. Budil, J. Tang, C.A. Wraight, and G.L. Closs, Proc. Nat. Acad. Sci., 79(1982)5532; (c) M.R. Wasielewski, J.R. Norris, and M.K. Bowman, Faraday Disc. Chem. Soc., 78(1984)279; (d) M. Okazaki, S. Sakata, R. Konaka, and T. Shiga, J. Chem. Phys., 86(1987)6972.

59. (a) E.L. Frakevich, A.I. Pristupa, JETP Lett., 24(1976)362, translated from Prisma Zh. Eksp. Teor. Fiz., 24(1976)397; (b) E.L. Frankevic, A.I. Pristupa, and V.I. Lesin, Chem. Phys. Lett., 47(1977)304.

60. (a) G.L. Closs and C.D. Doubleday, Jr., J. Am. Chem. Soc., 95(1973)2753;

(b) G.L. Closs, in L.P. Muus, P.W. Atkins, and J.B. Pedersen, (Eds.), Chemically Induced Magnetic Polarization, Reidel, Dordrecht, Holland, 1977.

61. C. Doubleday, Jr., N.J. Turro, and J.-F. Wang, Acc. Chem. Res., 22(1989) 199 and references within.

62. M.B. Zimmt, C.D. Doubleday, Jr., and N.J. Turro, J. Am. Chem. Soc., $106(1984) 3363$.

63. J.F. Wang, K.M. Welsh, K.C. Waterman, P. Fehlner, C.D. Doubleday, Jr., and N.J. Turro, J. Phys. Chem., 92(1988)3730.

64. (a) M.V. Encinas and J.C. Scaiano, J. Photochem., 11(1979)241; (b) J.C. Scaiano, Tetrahedron, 38(1982)819.

65. R.A. Caldwell and D. Creed, J. Phys. Chem,, 25(1978)2644.

66. J.C. Scaiano, Chem. Phys. Lett., 79(1981)441.

67. V.A. Kuzmin, and A.S. Tatikolov, Chem. Phys. Lett., 51(1981)441.

68. S. Sinha, Complexes of Rare Earths, Pergamon, Oxford, 1966, chapter 1.

69. D. Wayner, D. McPhee, and D. Griller, J. Am. Chem. Soc., 110(1988)132.

70. The total angular momentum is usually represented by the letter $\mathrm{J}$, but we have chosen another letter to avoid confusion.

71. Y. Sakaguchi and H. Hayashi, Chem. Phys. Lett., 106(1984)420.

72. N.J. Turro, X. Lei, I.R. Gould, and M.B. Zimmt, Chem. Phys. Lett., $120(1985) 397$.

73. (a) S. Basu, D. Nath, and M. Chowdhury, J. Luminescence 40(1988)252 and 41(1988)252; (b) S. Basu, L. Kundu, and M. Chowdhury, Chem. Phys. Lett., 141(1987)115. 
74. S. Basu; Ph.D Thesis, Jadavpur University: Calcutta, India 700032, 1989.

75. H. Hayashi and S. Nakagura, Bull. Chem. Soc. Jpn., 57(1984)322.

76. K. Obi and T. Imamura, Rev. Chem. Int., 7(1986)225 and references within.

77. N. J. Turro, Modern Molecular Photochemistry, Benjamin/Cummings, Menlo Park, CA, 1978, chapter 9.

78. T. Förster, Fluorenzenz Organische Verbindungen, Gottingen: Vandenhoech and Ruprech, 1951.

79. D.L. Dexter, J. Chem. Phys., 21(1953)836.

80. K. Akiyama, S. Tero-Kubota, T. Ikenoue, and Y. Ikegami, Chem. Lett., (1984)903.

81. K. Akiyama, S. Tero-Kubota, Y. Ikegami, and T. Ikenoue, J. Am. Chem. Soc., 106(1984)8322.

82. D. Weir and J.K.S. Wan, J. Am. Chem. Soc., 106(1984)427.

83. T. Imamura, O. Onitsuka, and K. Obi, J. Phys. Chem., 90(1986)6741.

84. A.I. Grant and K.A. McLauchlan, Chem. Phys. Lett., 101(1983)120.

85. $\mathrm{E} / \mathrm{A}^{*}$ means that and $\mathrm{E} / \mathrm{A}$ pattern is superposed over a net $\mathrm{A}$ polarization.

86. (a) V.A. Kuzmin and A.S. Tatikolov, Chem. Phys. Lett., 53(1978)606;

(b) S.K. Chattopadhyay, P.K. Das, and G.L. Hug, J. Am. Chem. Soc., $105(1983) 6205$.

87. A.R. Watkins, Chem. Phys. Lett., 70(1980)262.

88. (a) K.R. Naqvi and U.P. Wild, Chem. Phys. Lett., 41(1976)570; (b) K. Bhattacharyya, P.K. Das, R.W. Fessenden, M.V. George, K.R. Gopidas, and G. L. Hug, J. Phys. Chem., 89(1985)4164.

89. N.N. Quan and A.V. Guzzo, J. Phys. Chem., 85(1981)140.

90. K.R. Naqvi, H. Staerk, and T. Gillbro, Chem. Phys. Lett., 49(1977)160.

91. W.S. Jenks and N.J. Turro, unpublished results.

92. W.S. Jenks and N.J. Turro, Tetrahedron Lett., 30(1989)4469; There is a typographical error in Figure 1 of this paper. The scale should read $10 \mathrm{G}$ instead of $15 \mathrm{G}$.

93. (a) L.T. Calcaterrra, G.L. Closs, J.R. Miller J. Am. Chem. Soc., 105(1983) 670; (b) J.R. Miller, L.T. Calcaterrra, and G.L. Closs, J. Am. Chem. Soc., 106(1984)3047; (c) G.L. Closs, L.T. Calcaterrra, N.J. Green, K.W. Penfield, and J.R. Miller, J. Phys. Chem., 90(1986)3673; (d) G.L. Closs and J.R. Miller, Science, 240(1988)440.

94. G.L. Closs, P. Piotrowiak, J.M. MacInnis, and G. R. Fleming, J. Am. Chem. Soc., 110(1988)2652.

95. M.D. Miller, J.R. Miller, N.D. Green, and G.L. Closs, J. Phys. Chem., 93(1989) 1173.

96. G.L. Closs, M.D. Johnson, J.R. Miller, and P. Piotrowiak, J. Am. Chem. Soc., 111(1989) 3751 . 
97. M.T. Craw, M.C. Depew, and J.K.S. Wan, Rev. Chem. Int., 7(1986)215.

98. H.C. Torrey, Phys. Rev., 76(1949)1059.

99. I. Charmichael and H. Paul, Chem. Phys. Lett., 67(1979)519.

100. S. Basu, A.I. Grant, and K.A. McLauchlan, Chem. Phys. Lett., 94(1983)517.

101. A.I. Grant, N.J.B. Green, P.J. Hore, and K. A. McLauchlan, Chem. Phys. Lett., 110(1984)280.

102. F. Jent, H. Paul, K.A. McLauchlan, and D.G. Stephens, Chem. Phys. Lett., 141(1987) 443.

103. M.C. Thurnauer, T.-K. Chiu, and A.D. Trifunac, Chem. Phys. Lett., $116(1985) 543$.

104. K.A. McLauchlan, personal communication.

105. L.T. Muus, Chem. Phys. Lett., 160(1989)17.

106. V.I. Valyaev, Y.N. Molin, R.Z. Sagdeev, P.J. Hore, K.A. McLauchlan, and N.J.K. Simpson, Mol. Phys, 63(1988)891.

107. a) F. Jent and H. Paul, Chem. Phys. Lett., 160(1989)632; b) P.P. Borbat, A.D. Milov, and Yu. Molin, Chem. Phys. Lett., 164(1989)330.

108. Spin exchange between excited triplets and TEMPO in the absence of energy transfer is probably too slow for efficient spin polarization transfer. There is little reason to anticipate that its rate should follow the pattern of Figure 19. See, for instance, V.A. Kuzmin and A.S. Tatikolov, Chem. Phys. Lett., 53(1978)606 and A.R. Watkins, Chem. Phys. Lett., 70(1980)262.

109. D. Griller, L.M. Hadel, A.S. Nazran, M.S. Platz, P.C. Wong, and J.C. Scaiano, J. Am. Chem. Soc. 106(1984)2227.

110. (a) T.G. Savino, V.P. Senthilnathan, and M.S. Platz, Tetrahedron, 42(1986)2167; (b) D. Griller, C.R. Montgomery, J.C. Scaiano, M.S. Platz, and L.M. Hadel, J. Am. Chem. Soc., 104(1982)6813; (c) B.-E. Brauer, P.B. Grasse, K.J. Kaufmann, and G.B. Schuster, J. Am. Chem. Soc., 104(1982)6814; (d) P.B. Grasse, B.-E. Brauer, J.J. Zupancic, K.J. Kaufmann, and G.B. Schuster, J. Am. Chem. Soc., 105(1983)6833.

111. E. Kotsch, Z. Nat., 26a(1971)1836.

112. H.L. Casal, N.H. Werstiuk, and J.C. Scaiano, J. Org. Chem., 49(1984)5214.

113. J. Chateauneuf, J. Lusztyk, and K.U. Ingold, J. Org. Chem., 53(1988)1629.

114. Y. Sakaguchi, H. Hayashi, H. Murai, Y.J. I'Haya, and K. Mochida, Chem. Phys. Lett. 120(1985) 401.

115. Y. Sakaguchi and H. Hayashi, J. Phys. Chem., 90(1986)550.

116. K.A. McLauchlan and A.J.D. Ritchie, Chem. Soc. Perkin. Trans. II, (1984) 275 .

117. a) F. Jent, H. Paul, and H. Fischer, Chem. Phys. Lett., 146(1988)315; b) P. Jaegerman, F. Lendzian, G. Rist, and K. Möbius, Chem. Phys. Lett., $140(1987) 615$. 
118. P.P. Bordat and Y.D. Tsvetkov, Doklady Phys. Chem., 278(1985)865, translated from Doklady Adademii Nauk SSSR, 4(1984)900.

119. R. Baltzly, N.B. Mehta, P.B. Russell, R.E. Brooks, E.M. Grivsky, and A.M. Steinberg, J. Org. Chem., 26(1961)3669.

120. N.J. Turro, M. Paczkowski, M.B. Zimmt, and J.K.S. Wan, Chem. Phys. Lett., 114(1985)561.

121. (a) H. Paul, Chem. Phys., 40(1979)265; (b) S.K. Wong, T.M. Chiu, and J.R. Bolton, J. Phys. Chem., 85(1981)12; (c) H. Paul, J. Phys. Chem., 86(1982)4086; (d) S. Basu, A.I. Grant, and K.A. McLauchlan, Chem. Phys. Lett., 94(1983)517: (e) A.I. Grant, N.J.P. Green, P.J. Hore, and K. A. McLauchlan, Chem. Phys. Lett., 110(1984)280; (f) M.C. Thurnauer, T.-M. Chiu, and A.D. Trifunac. Chem. Phys. Lett., 116(1985)543; (g) S. Yamauchi, K. Tominaga, and N. Hirota, J. Phys. Chem., 90(1986)2369; (h) C.D. Buckley, D.A. Hunter, P.J. Hore, and K.A. McLauchlan, Chem. Phys. Lett., 135(1987)307; (i) K. Tominaga, S. Yamauchi, and N. Hirota, J. Chem. Phys., 88(1988)533.

122. M.B. Zimmt, C. Doubleday, Jr, and N.J. Turro, J. Am. Chem. Soc., $108(1986) 3618$. 